\title{
Nonnegative Periodic Solutions of a Three-Term Recurrence Relation Depending on Two Real Parameters
}

\author{
Yen Chih Chang, ${ }^{1}$ Sui Sun Cheng, ${ }^{2}$ and Wei Chang Yeh ${ }^{1}$ \\ ${ }^{1}$ Department of Industrial Engineering and Industrial Management, National Tsing Hua University, Hsinchu 30013, Taiwan \\ ${ }^{2}$ Department of Mathematics, National Tsing Hua University, Hsinchu 30013, Taiwan \\ Correspondence should be addressed to Sui Sun Cheng; sscheng@math.nthu.edu.tw
}

Received 30 October 2016; Accepted 7 February 2017; Published 6 September 2017

Academic Editor: Zhan Zhou

Copyright (c) 2017 Yen Chih Chang et al. This is an open access article distributed under the Creative Commons Attribution License, which permits unrestricted use, distribution, and reproduction in any medium, provided the original work is properly cited.

\begin{abstract}
Simple dynamic systems representing time varying states of interconnected neurons may exhibit extremely complex behaviors when bifurcation parameters are switched from one set of values to another. In this paper, motivated by simulation results, we examine the steady states of one such system with bang-bang control and two real parameters. We found that nonnegative and negative periodic states are of special interests since these states are solutions of linear nonhomogeneous three-term recurrence relations. Although the standard approach to analyse such recurrence relations is the method of finding the general solutions by means of variation of parameters, we find novel alternate geometric methods that offer the tracking of solution trajectories in the plane. By means of this geometric approach, we are then able, without much tedious computation, to completely characterize the nonnegative and negative periodic solutions in terms of the bifurcation parameters.
\end{abstract}

\section{Introduction}

Simple dynamic systems representing time varying states of interconnected compartments or "neurons" may exhibit extremely complex behaviors when bifurcation parameters are switched from one set of values to another. An example has been given in several of our previous studies [1] and a slightly modified model of which is described as follows. Let $n$ neurons be placed on the vertices of a regular $n$-gon and let the time dependent state values of the neurons be denoted by $u_{i}(t)$, for $i=1,2, \ldots, n$. Suppose the rate of change $u_{2}^{\prime}(t)$ is determined by the resultant effect of a constant multiple of $u_{2}(t)$, plus a magnified on-off (or bang bang) state dependent control mechanism as well as its two near neighbors $u_{1}(t)$, $u_{3}(t)$ in the form

$$
u_{2}^{\prime}(t)=u_{1}(t)+\rho u_{2}(t)+u_{2}(t)-\mu H\left(u_{2}(t)\right) .
$$

Then assuming "uniformity" among the neuron interactions, we have

$$
\begin{array}{r}
u_{i}^{\prime}(t)=u_{i-1}(t)+\rho u_{i}(t)+u_{i+1}(t)-\mu H\left(u_{i}(t)\right), \\
i=1,2, \ldots, n,
\end{array}
$$

where $u_{0}(t)$ is identified with $u_{n-1}(t)$ while $u_{n+1}(t)$ with $u_{1}(t)$ for compatibility reasons. Here $H(x)$ is the on-off Heaviside step function defined by

$$
H(x)= \begin{cases}1 & \text { if } x \geq 0 \\ -1 & \text { if } x<0 .\end{cases}
$$

When the real parameters $\rho$ and $\mu$ are switched among different real values, it is expected (and verified by simulations) that complex dynamic behaviors will be more than abundant. Some of these behaviors can be explained (see [13]) but some not (at least to the best of our knowledge). Similar models of piecewise constant dynamic systems which exhibit similar behaviors with parameters can be found in many recent investigations; see for examples [4-10] and the references therein.

In this note, we discuss the steady state solutions of the above dynamic system (i.e., those that satisfy $u_{k}(t) \equiv \varphi_{k}$ for all $t$ and for each $k=1,2, \ldots$, or $n)$. Then we will face 
the existence problem of periodic solutions of the following three-term recurrence relation:

$$
\begin{aligned}
\varphi_{k}+\rho \varphi_{k+1}+\varphi_{k+2}=\mu H\left(\varphi_{k+1}\right) & \\
\qquad & \quad k \in \mathbf{Z}:=\{\ldots,-1,0,1, \ldots\} .
\end{aligned}
$$

In [1-3], we are lucky to obtain complete information about the periodic solutions of (4) when $\mu=1$ and $\rho=1,0$, or -1 by breaking the solutions into two sequences, a companion and an error (more specifically, for a solution $\varphi=\left\{\varphi_{k}\right\}_{k \in \mathbf{N}}$ of (4) with $\mu=1$ and $\rho \in\{-1,0,1\}$, the companion sequence is $\lambda=\left\{\lambda_{k}\right\}_{k \in \mathbf{N}}$ which is an even integral sequence so that $\lambda_{k}=$ $2\left[(1 / 2) \varphi_{k}\right]$, where $2[x / 2]$ is the greatest even integer that is less than or equal to $x \in \mathbf{R}$, and the error sequence $\epsilon=\left\{\epsilon_{k}\right\}_{k \in \mathbf{N}}$ is defined by $\epsilon_{k}=\varphi_{k}-\lambda_{k}, k \in \mathbf{Z}$ ).

Yet, for other values of $\mu$ and $\rho$, simulations show complex periodic behaviors beyond our present comprehension, except when the periodic solutions are also "nonnegative" (or "negative"). Such exceptional results, when examined more closely, can be explained.

In this paper, we will devote ourselves to explaining the behaviors of these nonnegative (or negative) and periodic solutions of (7).

First, a real sequence $\varphi=\left\{\varphi_{k}\right\}_{k \in \mathbf{Z}}$ is a solution of (4) if it renders (4) into an identity after substitution. It is nonnegative (or negative) if all its terms are nonnegative (respectively negative). If $\zeta=\left\{\zeta_{k}\right\}_{k \in \mathbf{Z}}$ is a nonnegative solution of (4), then clearly $\zeta$ satisfies

$$
\zeta_{m}+\rho \zeta_{m+1}+\zeta_{m+2}=\mu, \quad m \in \mathbf{Z},
$$

while if $\xi$ is a negative solution of (4), then

$$
\xi_{n}+\rho \xi_{n+1}+\xi_{n+2}=-\mu, \quad n \in \mathbf{Z} .
$$

Hence it seems that we are back to the usual nonhomogeneous linear second-order difference equations studied in elementary theory of difference equations. Since $\mu \in \mathbf{R}$ in (5) and (6), we can further restrict ourselves in the sequel to the following difference equation:

$$
\varphi_{k}+\rho \varphi_{k+1}+\varphi_{k+2}=\mu, \quad \mu \in \mathbf{R}, k \in \mathbf{Z},
$$

with arbitrary $\mu \in \mathbf{R}$. By means of the techniques of general solutions plus the method of variation of parameters, it may be argued that the existence of nonnegative (respectively negative) periodic solutions can be handled completely. Such an assertion may be true in theory, but the general solution here is $\rho$ - as well as $\mu$-dependent and hence actual attempts lead to many complications.

That said, in this paper, we will handle our equation from a novel approach and the crux of which is based on representing each pair of two consecutive terms $\left(\varphi_{k}, \varphi_{k+1}\right)$ of a solution $\varphi=\left\{\varphi_{k}\right\}_{k \in Z}$ as a point in the plane and invent a geometric method to track the movements of these points. First, the totality of such pairs is called the orbit of this solution. More precisely, let $\varphi=\left\{\varphi_{k}\right\}_{k \in \mathbf{Z}}$ be a solution of (4). We define the orbit of $\varphi$ by

$$
\mathcal{O}(\varphi):=\left\{\left(x_{k}, y_{k}\right) \in \mathbf{R}^{2} \mid x_{k}=\varphi_{k}, y_{k}=\varphi_{k+1}, k \in \mathbf{Z}\right\}
$$

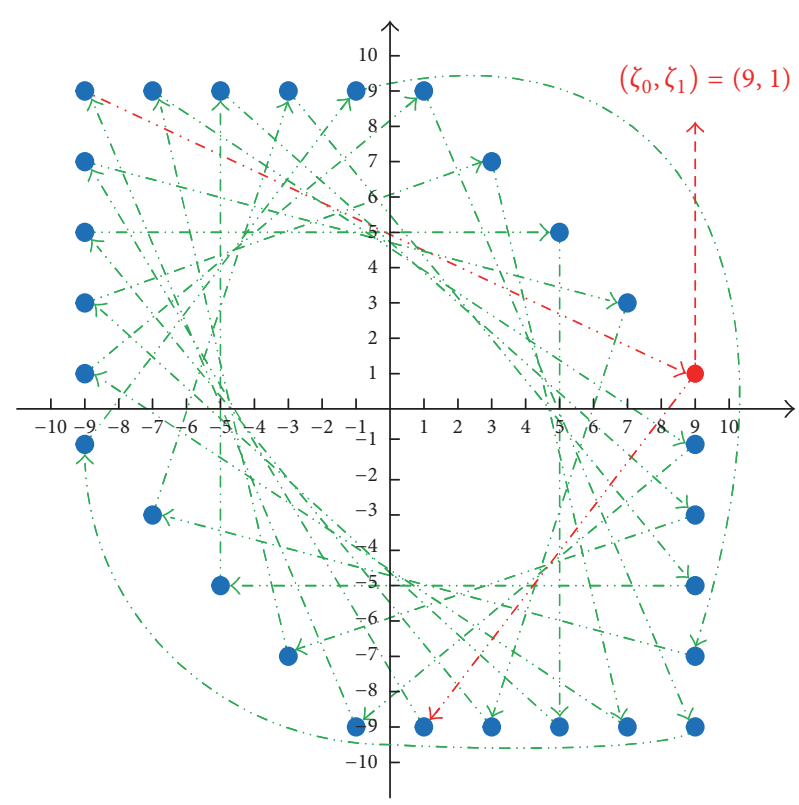

Figure 1: The orbit of $\zeta$ with $\rho=1$.

and the "positive" orbit by

$$
\begin{aligned}
& \mathcal{O}^{+}(\varphi) \\
& :=\left\{\left(x_{k}, y_{k}\right) \in \mathbf{R}^{2} \mid x_{k}=\varphi_{k}, y_{k}=\varphi_{k+1}, k \in \mathbf{N}\right\}, \\
& \mathbf{N}:=\{0,1,2, \ldots\} .
\end{aligned}
$$

As examples, let $\mu=1$ and let $\zeta$ and $\xi$ be solutions of (4) with $\rho=1$ or 0 , respectively. The orbits $\mathcal{O}(\zeta)$ with $\left(\zeta_{0}, \zeta_{1}\right)=$ $(9,1)$ and $\mathcal{O}(\xi)$ with $\left(\xi_{0}, \xi_{1}\right)=(15 / 2,1 / 2)$ are depicted in Figures 1 and 2 , respectively. These figures clearly suggest that $\zeta$ is " 28 -periodic," that $\xi$ is " 30 -periodic," and that there are accompanying "distinctive" features which can be exploited further.

Several sets will be encountered in the ensuing discussions and we denote them as follows:

(i) $\mathbf{R}:=(-\infty, \infty)$ is the entire set of real numbers.

(ii) $\mathbf{Z}:=\{\ldots,-1,0,1, \ldots\}$ is the set of integers

(iii) $\mathbf{Z}^{+}:=\{1,2,3, \ldots\}$ is the set of positive integers.

(iv) $\mathbf{N}:=\{0,1,2, \ldots\}$ is the set of nonnegative integers.

(v) $\mathbf{Q}:=\left\{ \pm m / n \mid m \in \mathbf{N}, n \in \mathbf{Z}^{+}\right\}$is the set of rational numbers.

Before we enter into discussions on the necessary and sufficient conditions for a solution of (7) to be nonnegative and periodic, we take a note that any solution $\varphi=\left\{\varphi_{k}\right\}_{k \in \mathbf{Z}}$ of our three-term equation (7) is uniquely determined by two of its consecutive terms. Furthermore, in view of the fact that (7) can be written as

$$
\varphi_{m-1}+\varphi_{m+1}=\mu-\rho \varphi_{m}, \quad m \in \mathbf{Z},
$$

as well as

$$
\varphi_{m+1}+\rho \varphi_{m+2}+\varphi_{m+3}=\mu, \quad m \in \mathbf{Z},
$$




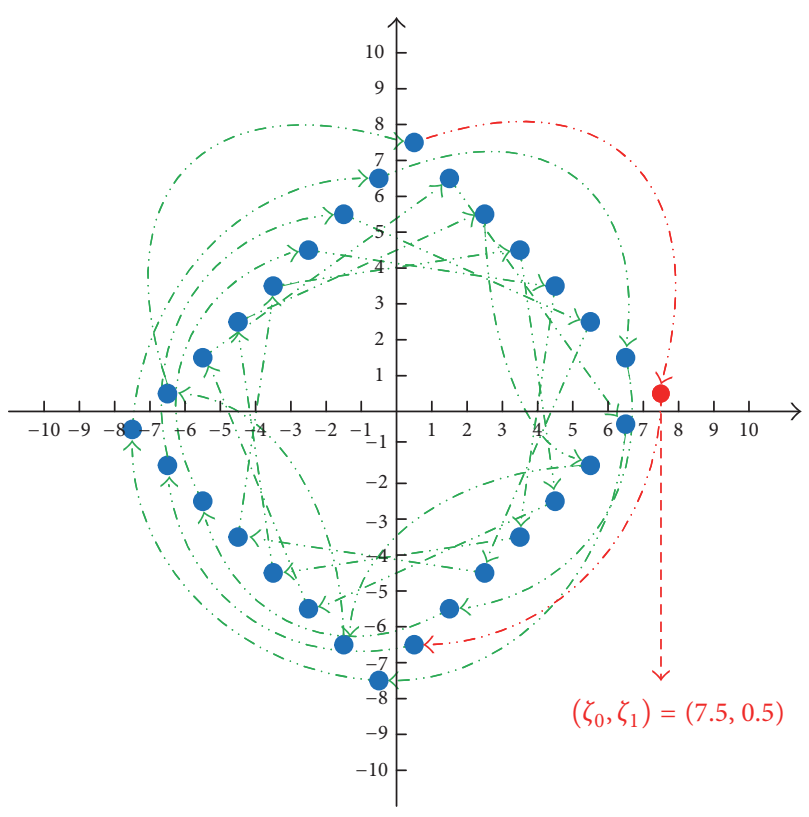

FIgure 2: The orbit of $\xi$ with $\rho=0$.

it is both "symmetric" (or "reflection invariant") and "translation invariant." More precisely, let $\left\{\varphi_{j}\right\}_{j \in \mathbf{Z}}$ be the solution of (7) that satisfies $\varphi_{0}=\alpha$ and $\varphi_{1}=\beta$. Then the solution $\left\{\xi_{j}\right\}_{j \in \mathbf{Z}}$ of (7) determined by $\xi_{0}=\alpha$ and $\xi_{-1}=\beta$, called the reflection of $\varphi$ and denoted by $\xi=U \varphi$, will satisfy

$$
\xi_{j}=\varphi_{-j}, \quad j \in \mathbf{Z},
$$

while the solution $\left\{\zeta_{j}\right\}_{j \in \mathbf{Z}}$ of (7) determined by $\zeta_{k}=\alpha$ and $\zeta_{k+1}=\beta$ for any $k \in \mathbf{Z}$, called the $k$-translation of $\varphi$ and denoted by $\zeta=E^{k} \varphi$, will satisfy

$$
\zeta_{j}=\varphi_{j-k}, \quad k \in \mathbf{Z}
$$

In view of these invariances, to study (7), we may simply concentrate our attention on the positive orbits of its solutions! Indeed, let $\psi=\left\{\psi_{k}\right\}_{k \in \mathbf{Z}}$ be the solution of (7) with $\left(\psi_{0}, \psi_{1}\right)=(\sigma, \tau)$. We investigate $\left\{\psi_{0}, \psi_{1}, \psi_{2}, \ldots\right\}$ by observing the positive orbit $\mathcal{O}^{+}(\psi)$ defined by $(\sigma, \tau)$. On the other hand, we analyze $\left\{\psi_{1}, \psi_{0}, \psi_{-1}, \psi_{-2}, \ldots\right\}$ by studying the positive orbit $\mathcal{O}^{+}\left(U E^{-1} \psi\right)$. Note that for all $\left(\psi_{n}, \psi_{n+1}\right) \in \mathcal{O}(\psi)$, where $n<0$, $\left(\psi_{n+1}, \psi_{n}\right)=Q_{|n|}$, where $Q_{|n|} \in \mathcal{O}^{+}\left(U E^{-1} \psi\right)$. In the sequel, if no doubt arises, we frequently let $\varphi=\left\{\varphi_{k}\right\}_{k \in \mathbf{Z}}$ be a solution of (7) which is defined by $\left(\varphi_{0}, \varphi_{1}\right)=(\alpha, \beta)$ and we denote

$$
\begin{aligned}
& \mathcal{O}_{\perp}^{+}(\varphi)=\left\{\left(x_{k}, y_{k}\right)=\left(v_{k+1}, u_{k+1}\right) \mid\left(u_{k+1}, v_{k+1}\right)\right. \\
& \left.\quad \in \mathcal{O}^{+}\left(U E^{-1} \varphi\right), k \in \mathbf{N}\right\}
\end{aligned}
$$

so that

$$
\mathcal{O}(\varphi)=\mathcal{O}^{+}(\varphi) \cup \mathcal{O}_{\perp}^{+}(\varphi)
$$

Proposition 1. Let $\varphi=\left\{\varphi_{k}\right\}_{k \in \mathbf{Z}}$ be a solution of (7) defined by $\left(\varphi_{0}, \varphi_{1}\right)=(\alpha, \beta)$ and $\mathcal{O}^{+}(\varphi)=\left\{\left(x_{k}, y_{k}\right)_{k}\right\}_{k \in \mathbf{N}}$ be the "positive" orbit of $\varphi$. Then $\omega$ is a period of $\varphi$ if and only if $\left(x_{\omega}, y_{\omega}\right)=$ $(\alpha, \beta)$. Furthermore, if $\lim _{k \rightarrow \infty}\left|x_{k}\right|=\infty$, then $\varphi$ is aperiodic and if $\lim _{k \rightarrow \infty} x_{k} y_{k}<0$, then $\varphi$ is neither a nonnegative nor a negative solution.

The statements of Proposition 1 can be verified directly from the definitions, as well as the invariant properties of (7), and, thus, the proof is omitted here.

\section{Some Basic Results of (7)}

Periods and the prime periods of sequences are defined as usual. Furthermore, let $\varphi=\left\{\varphi_{k}\right\}_{k \in \mathbf{Z}}$ be a nonnegative solution of (7). Note that for some distinct $m, n \in \mathbf{N}$, if $\left(\varphi_{m}, \varphi_{m+1}\right)=$ $\left(\varphi_{n}, \varphi_{n+1}\right)$, then $|m-n|$ is a period of $\varphi$ and the least one $\omega$ among the periods is the least or prime period of $\varphi$ (we also say that $\varphi$ is $\omega$-periodic). A constant solution of (7) is 1periodic. We first find the necessary and sufficient conditions for $\varphi$ to be a constant solution.

Proposition 2. Let $\varphi=\left\{\varphi_{m}\right\}_{m \in \mathbf{Z}}$ be a solution of (7) with $\left(\varphi_{0}, \varphi_{1}\right)=(\alpha, \beta)$.

(i) Suppose $\rho \neq-2$. Then $\varphi$ is a constant solution if and only if $\alpha=\beta=\mu /(2+\rho)$.

(ii) Suppose $\rho=-2$. Then $\varphi$ is a constant solution if and only if $\mu=0$ and $\alpha=\beta$.

Proof. Suppose $\rho \neq-2$. If $\varphi$ is a constant solution of (7), then $\alpha=\beta=\varphi_{2}$ and

$$
\varphi_{2}=\alpha=\mu-\rho \varphi_{1}-\varphi_{0}=\mu-\rho \beta-\alpha=\mu-\rho \alpha-\alpha
$$

so that

$$
\alpha(2+\rho)=\mu \text {. }
$$

Accordingly, $\alpha=\mu /(2+\rho)=\beta$. The converse can be verified by direct iteration. Next, suppose $\rho=-2$. If $\varphi$ is a constant solution of (7), then $\alpha=\beta$ and by (7)

$$
\varphi_{2}=\mu+2 \beta-\alpha=\mu+\alpha=\alpha
$$

which leads to $\mu=0$. Conversely, if $\mu=0$ and $\alpha=\beta$, then by iteration $\varphi$ is clearly a constant solution.

The proof is complete.

Corollary 3. Let $\varphi=\left\{\varphi_{k}\right\}_{k \in \mathbf{Z}}$ be a solution of (7) with $\varphi_{0}=$ $\varphi_{1}=\gamma$. Let $\gamma=\mu /(2+\rho)$ where $\rho \neq-2$. Then $\varphi$ is 1-periodic. $\varphi$ is a nonnegative solution if and only if $\gamma \geq 0$; and $\varphi$ is a negative solution if and only if $\gamma<0$.

The results can be obtained from Proposition 2 directly and, hence, we omit the proof.

In view of Proposition 2 , it is easy to see that the orbit $\mathcal{O}(\varphi)$ of a constant solution is just the set containing the only point $(\mu /(2+\rho), \mu /(2+\rho))$

Proposition 4. Let $\varphi=\left\{\varphi_{k}\right\}_{k \in \mathrm{Z}}$ be a solution of (5) with $\left(\varphi_{0}\right.$, $\left.\varphi_{1}\right)=(\alpha, \beta)$. Then $\varphi$ is 2-periodic if and only if $\rho=2, \alpha+\beta=$ $\mu / 2$, and $\alpha \neq \beta$. 
Proof. Suppose $\varphi$ is 2-periodic. Then $\varphi_{2}=\alpha$ as well as $\varphi_{3}=\beta$ and by (5), $\varphi_{2}=\mu-\rho \beta-\alpha=\alpha$ and $\varphi_{3}=\mu-\rho \alpha-\beta=\beta$ which lead us to $2 \alpha+\rho \beta=\mu$ and $\rho \alpha+2 \beta=\mu$, respectively. Accordingly, we have $(2+\rho)(\alpha+\beta)=2 \mu$ and $(2-\rho)(\alpha-\beta)=0$. Note that since $\varphi$ is 2-periodic, we can be sure that $\alpha \neq \beta$. Hence, if $\rho \neq 2$, then $\alpha-\beta=0$ which is a contradiction; if $\rho=2$, then $\alpha+\beta=\mu / 2$ and $\alpha \neq \beta$. The converse may be checked by direct substitution into (5) and this completes the proof.

Corollary 5. Let $\varphi=\left\{\varphi_{k}\right\}_{k \in \mathbf{Z}}$ be a solution of (7) with $\rho=2$ and $\left(\varphi_{0}, \varphi_{1}\right)=(\alpha, \beta)$. Suppose $\alpha+\beta=\mu / 2$. Then $\varphi$ is periodic with period 2. $\varphi$ is nonnegative if and only if $\alpha, \beta, \mu \geq 0$; and $\varphi$ is negative if and only if $\alpha, \beta, \mu<0$.

Proof. Suppose $\alpha+\beta=\mu / 2$. First of all, if $\alpha=\beta=\mu / 4$, then, by Proposition 2, $\varphi$ is 1-periodic; otherwise, by Proposition 4, $\varphi$ is 2-periodic. Hence, $\varphi$ is periodic with period 2. If $\varphi$ is a nonnegative solution, then $\alpha, \beta \geq 0$ which implies $\mu \geq 0$. Furthermore, since $\varphi$ is periodic with period 2, we can see that $\varphi_{k} \geq 0$ for all $k \in \mathbf{Z}$. If $\alpha, \beta, \mu \geq 0$, then $\alpha+\beta=\mu / 2$ is satisfied and $\varphi$ is a nonnegative solution. The case where $\varphi$ is a negative solution can be handled similarly and this completes the proof.

In this paper, since we are interested in the periodicity of the solutions of (4) which are also nonnegative (or negative), the following results will be useful.

Proposition 6. Let $\varphi=\left\{\varphi_{k}\right\}_{k \in \mathbf{Z}}$ be a nonnull solution of (7). If $\mu \geq 0$ and $\rho \geq 0$, then $\varphi$ cannot be a nonpositive solution; if $\mu \leq 0$ and $\rho \leq 0$, then $\varphi$ cannot be a nonnegative solution.

Proof. Suppose $\mu \geq 0$ and $\rho \geq 0$. If $\varphi$ is nonpositive, then $0 \geq \varphi_{2}=-\rho \varphi_{1}-\varphi_{0}+\mu \geq 0$ and $0 \geq \varphi_{3}=-\rho \varphi_{2}-\varphi_{1}+\mu \geq 0$. Hence $\varphi_{2}=\varphi_{3}=0$, which implies $\varphi$ is null, contrary to our assumption.

Suppose $\mu \leq 0$ and $\rho \leq 0$. If $\varphi$ is nonnegative, then $0 \leq$ $\varphi_{2}=-\rho \varphi_{1}-\varphi_{0}+\mu \leq 0$ and $0 \leq \varphi_{3}=-\rho \varphi_{2}-\varphi_{1}+\mu \leq 0$ so that $\varphi_{2}=\varphi_{3}=0$. A contradiction is arrived and this completes the proof.

Proposition 7. $\varphi$ is a solution of (5) if and only if $-\varphi$ is a solution of (6).

Proof. Suppose that $\varphi=\left\{\varphi_{m}\right\}_{m \in \mathbf{Z}}$ is a solution of (5) with $\left(\varphi_{0}, \varphi_{1}\right)=(\alpha, \beta)$. By (5), we have $\varphi_{2}=\mu-\rho \beta-\alpha$. Let $\psi=-\varphi$ such that $\left(\psi_{0}, \psi_{1}\right)=(-\alpha,-\beta)$. Accordingly, $\psi_{2}=-\varphi_{2}$ and we see that

$$
\begin{aligned}
\psi_{2} & =-(\mu-\rho \beta-\alpha) \\
& =-\mu+\rho \beta+\alpha \\
& =-\mu-\rho \psi_{1}-\psi_{0}
\end{aligned}
$$

which satisfies (6). By induction, it follows that $\psi$ is a solution of (6) and this completes the proof.

Next, we discuss nonconstant solutions of (5) (although 2-periodic solutions are discussed in Proposition 4, we may

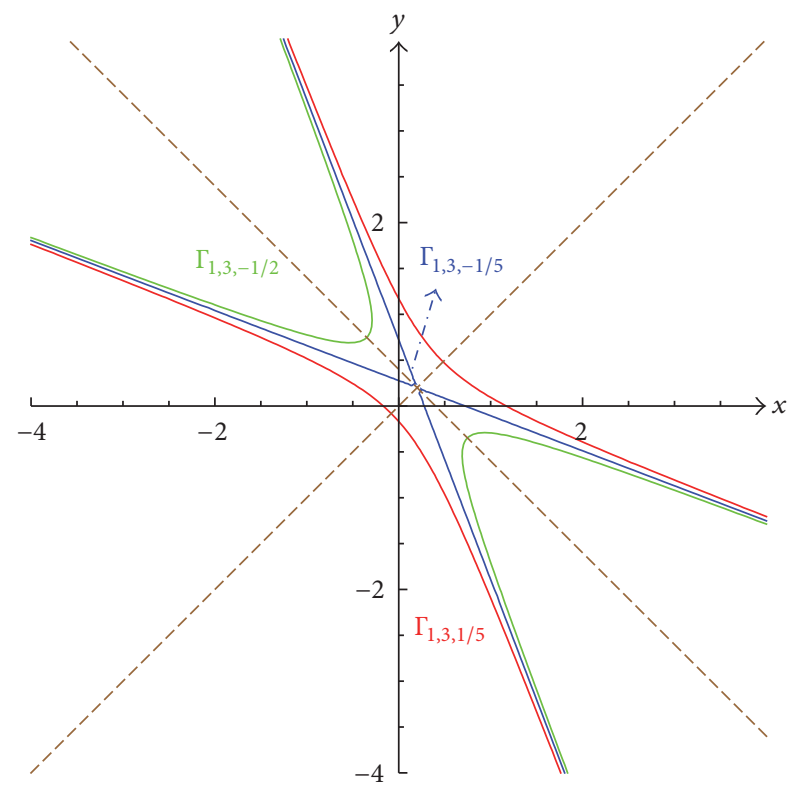

FIGURE 3: The hyperbolae with $\rho>2$.

include them in the following discussions). The behavior of solution $\varphi$ of (7) with least period 1 (or 2) was quite easy to analyze directly, yet it is difficult to conduct similar analyses for $\varphi$ with larger least period (or $\varphi$ is aperiodic) by similar manner. Hence, we will investigate the behavior of $\varphi$ from a new perspective.

\section{The Orbits of Solutions of (7)}

We will need the following quadratic function:

$$
\begin{aligned}
\Gamma_{\mu, \rho}(x, y)=x^{2}+\rho x y+y^{2}-\mu x-\mu y, & \\
& \text { where }(x, y) \in \mathbf{R}^{2},
\end{aligned}
$$

and its $f$-level curve $\Gamma_{\mu, \rho, f}$ is defined by

$$
\Gamma_{\mu, \rho, f}=\left\{(x, y) \in \mathbf{R}^{2} \mid \Gamma_{\mu, \rho}(x, y)=f, f \in \mathbf{R}\right\} .
$$

Since $\Gamma_{\mu, \rho}$ is a quadratic function, its level curves are plane conic sections. The properties of these conic sections are well known. First, we see that the $x=y$ line is one of the principal axes of $\Gamma_{\mu, \rho, f}$ and if $\rho \neq-2$; then $(\mu /(2+\rho), \mu /(2+\rho))$ is the center of $\Gamma_{\mu, \rho, f}$ and the other principal axis is the $x+y=2 \mu$ / $(2+\rho)$ line. In particular (see, e.g., [11]) $\Gamma_{\mu, \rho, f}$ can be classified by the value of its discriminant $\rho^{2}-4$ and the values $(\rho-2) \mu^{2}$ as well as $f$ :

(1) If $|\rho|>2$, then $\Gamma_{\mu, \rho, f}$ is a hyperbola (see the green and red curves in Figures 3 and 4$)$ when $f \neq-\mu^{2} /(2+\rho)$ or a degenerate hyperbola consisting of two intersecting lines when $f=-\mu^{2} /(2+\rho)$ (see the blue lines in Figures 3 and 4 ).

(2) If $|\rho|<2$, then $\Gamma_{\mu, \rho, f}$ is an ellipse (see Figure 5) when $f \neq-\mu^{2} /(2+\rho)$ or a degenerate point ellipse when $f=-\mu^{2} /(2+\rho)$. 


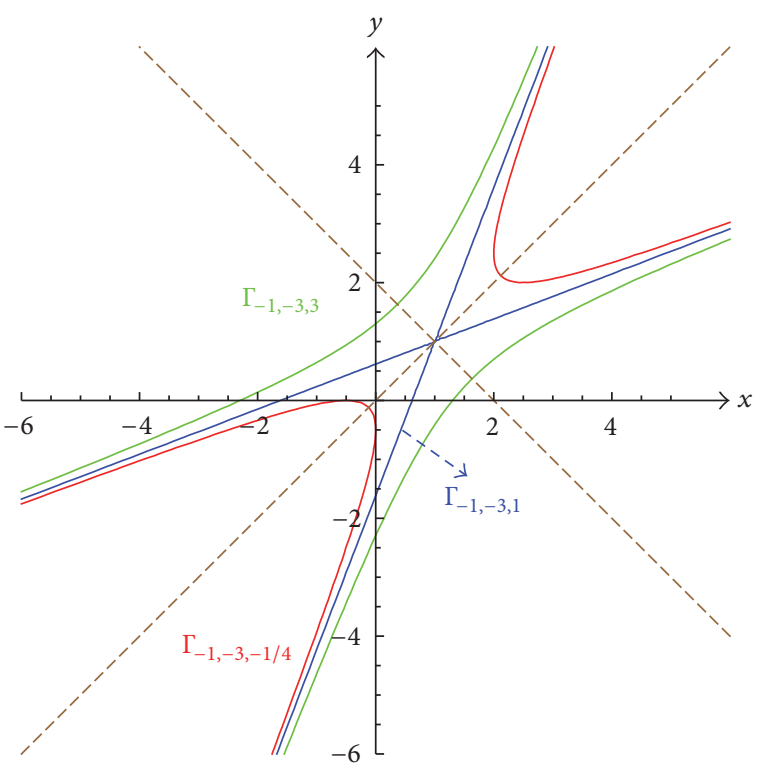

FIGURE 4: The hyperbolae with $\rho<-2$.

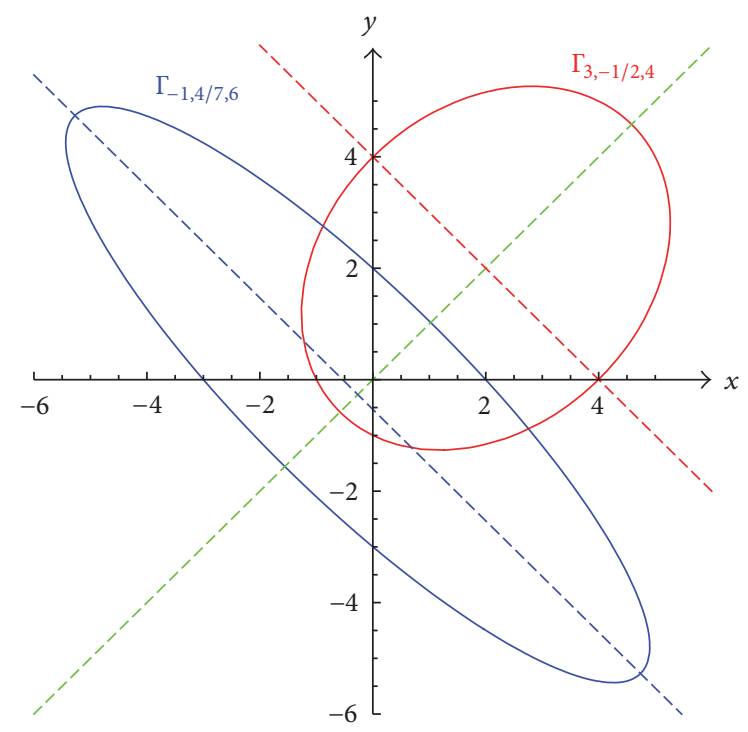

FIGURE 5: The ellipses with $-2<\rho<2$.

(3) If $|\rho|=2$, then $\Gamma_{\mu, \rho, f}$ is a parabola when $\rho=-2$ or a degenerate parabola consisting of a single or two distinct parallel lines when $\rho=2$ (see the green and blue lines in Figure 6).

Unless indicated by the adjective "degenerate," a conic section is meant to be nondegenerate. The conic sections $\Gamma_{\mu, \rho, f}$ are symmetric with respect to the $x=y$ line. This property is a simple consequence of the fact that $\Gamma_{\rho, f}(x, y)$ is a symmetric function in $x$ and $y$. Hence, the $x=y$ line is one of the principal axes of the conic sections.

To discuss the principal axes further, we define the two variable functions:

$$
\Pi(x, y)=x-y
$$

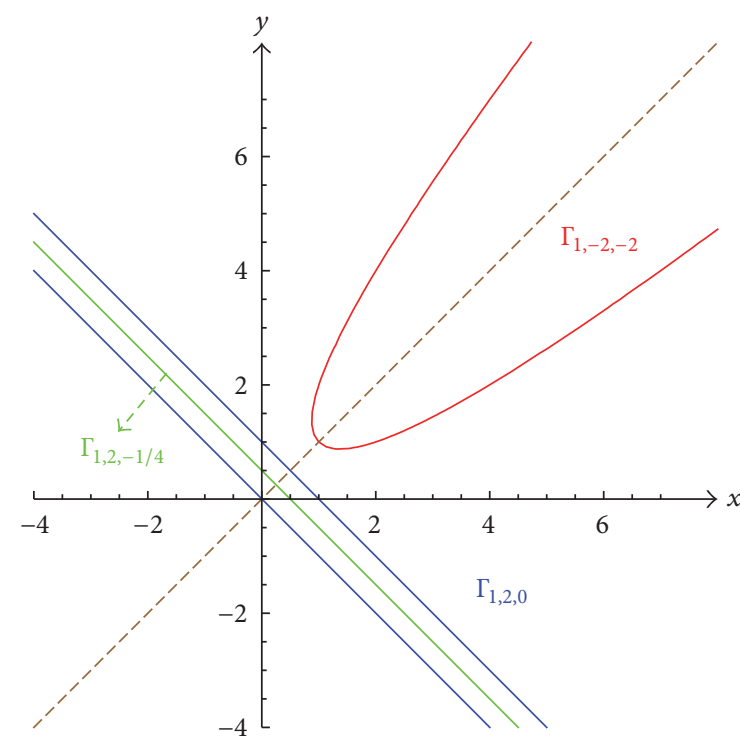

FIGURE 6: The parabolae with $|\rho|=2$.

and the associated line $\bar{\Pi}=\left\{(x, y) \in \mathbf{R}^{2} \mid \Pi(x, y)=0\right\}$. Also, for $\rho \neq-2$, we let

$$
\Pi_{\perp}(x, y)=x+y-\frac{2 \mu}{2+\rho}
$$

and $\bar{\Pi}_{\perp}=\left\{(x, y) \in \mathbf{R}^{2} \mid \Pi_{\perp}(x, y)=0\right\}$. Furthermore, let $f=\Gamma_{\mu, \rho}(\alpha, \beta)$, where $(\alpha, \beta) \in \mathbf{R}^{2}$ and $\delta=\sqrt{\rho^{2}-4}$ for $|\rho|>2$. We define the following two variable functions:

$$
\begin{aligned}
& L_{\mu, \rho, f}^{+}(x, y) \\
& \quad:= \begin{cases}y+\frac{1}{2}(\rho+\delta) x-\frac{\mu}{2}\left(1+\frac{\delta}{2+\rho}\right) & \text { if }|\rho|>2, \\
x+y-\frac{1}{2} \mu+\sqrt{f+\frac{1}{4} \mu^{2}} & \text { if } \rho=2, \\
x-y+\sqrt{f} & \text { if } \rho=-2,\end{cases}
\end{aligned}
$$

as well as

$$
\begin{aligned}
L_{\mu, \rho, f}^{-}(x, y) & \begin{cases}y+\frac{1}{2}(\rho-\delta) x-\frac{\mu}{2}\left(1-\frac{\delta}{2+\rho}\right) & \text { if }|\rho|>2, \\
x+y-\frac{1}{2} \mu-\sqrt{f+\frac{1}{4} \mu^{2}} & \text { if } \rho=2, \\
x-y-\sqrt{f} & \text { if } \rho=-2,\end{cases}
\end{aligned}
$$

with $\bar{L}_{\mu, \rho, f}^{+}=\left\{(x, y) \in \mathbf{R}^{2} \mid L_{\mu, \rho, f}^{+}(x, y)=0\right\}$ and $\bar{L}_{\mu, \rho, f}^{-}=$ $\left\{(x, y) \in \mathbf{R}^{2} \mid L_{\mu, \rho, f}^{-}(x, y)=0\right\}$. Note that in view of $(24)$ and (25), $L_{\mu, \rho, f}^{+}(x, y)$ as well as $L_{\mu, \rho, f}^{-}(x, y)$ are independent from $f$ when $|\rho|>2$.

\section{Let $S \subset \mathbf{R}^{2}$. Define}

$$
\begin{aligned}
S^{\prime} & =\left\{(x, y) \in \mathbf{R}^{2} \mid(y, x) \in S\right\}, \\
S^{\prime \prime} & =\left\{(x, y) \in \mathbf{R}^{2} \mid(-y,-x) \in S\right\} .
\end{aligned}
$$


Then $S^{\prime}$ and $S$ are symmetric with respect to the $x=y$ line, while $S^{\prime \prime}$ and $S$ are symmetric with respect to the $x+y=0$ line. The boundary of the plane set $S$ will be denoted by $\partial S$.

The conic sections will be of great help in the analysis of solutions of (4). The most significant result is the following.

Theorem 8. Let $\varphi=\left\{\varphi_{k}\right\}_{k \in \mathbf{Z}}$ be a solution of (5). If $f=$ $\Gamma_{\mu, \rho}\left(\varphi_{m}, \varphi_{m+1}\right)$ for some $m \in \mathrm{Z}$, then $\mathcal{O}(\varphi) \subseteq \Gamma_{\mu, \rho, f}$. Furthermore, if $(\alpha, \beta) \in \Gamma_{\mu, \rho, f}$, where $f=\Gamma_{\mu, \rho}(\alpha, \beta)$, then $(-\alpha,-\beta) \in \Gamma_{\mu, \rho, f}^{\prime \prime}$.

Proof. Without loss of generality, we let $m=0$ and $\left(\varphi_{0}, \varphi_{1}\right)=$ $(\alpha, \beta)$. Let $f=\Gamma_{\mu, \rho}(\alpha, \beta)$. Then $(\alpha, \beta) \in \Gamma_{\mu, \rho, f}$. By $(21)$, we see that

$$
\alpha^{2}+\rho \alpha \beta+\beta^{2}-\mu \alpha-\mu \beta-f=0
$$

and by (7), it follows $\left(\varphi_{1}, \varphi_{2}\right)=(\beta, \mu-\rho \beta-\alpha)$ so that

$$
\begin{aligned}
\Gamma_{\mu, \rho}(\beta, \mu-\rho \beta-\alpha)= & \beta^{2}+\beta(\mu-\rho \beta-\alpha) \rho \\
& +(\mu-\rho \beta-\alpha)^{2}-\beta \\
& -(\mu-\rho \beta-\alpha) \\
= & \alpha^{2}+\rho \alpha \beta+\beta^{2}-\mu \alpha-\mu \beta=f,
\end{aligned}
$$

which leads to $\left(\varphi_{1}, \varphi_{2}\right) \in \Gamma_{\mu, \rho, f}$. By induction, for any $n \in \mathbf{N}$, $\left(\varphi_{n}, \varphi_{n+1}\right) \in \Gamma_{\mu, \rho, f}$ which implies $\mathcal{O}^{+}(\varphi) \subseteq \Gamma_{\mu, \rho, f}$. Next, we consider $\left(\varphi_{n}, \varphi_{n+1}\right)$, where $n \in \mathbf{Z} \backslash \mathbf{N}$. Let $\psi \stackrel{\varphi_{k}}{=}\left\{\psi_{k}\right\}_{k \in \mathbf{Z}}=$ $U E^{-1} \varphi$ such that $\left(\psi_{0}, \psi_{1}\right)=(\beta, \alpha)$ and $\varphi_{n}=\psi_{-n+1}$. Since $\Gamma_{\mu, \rho, f}$ is symmetric with respect to the $x=y$ line, $(\beta, \alpha) \epsilon$ $\Gamma_{\mu, \rho, f}$ and by the previous discussions, we can be sure that $\mathcal{O}^{+}(\psi) \subset \Gamma_{\mu, \rho, f}$ which implies $Q_{n}^{\prime} \in \Gamma_{\mu, \rho, f}$, where $Q_{n} \in \mathcal{O}^{+}(\psi)$. Accordingly, $\mathcal{O}_{\perp}^{+}(\varphi) \subseteq \Gamma_{\mu, \rho, f}$ and by (15), it follows that $\mathcal{O}(\varphi) \subseteq$ $\Gamma_{\mu, \rho, f}$. Furthermore, by the definitions, if $(\alpha, \beta) \in \Gamma_{\mu, \rho, f}$, where $f=\Gamma_{\mu, \rho}(\alpha, \beta)$, then $(-\beta,-\alpha) \in \Gamma_{\mu, \rho, f}^{\prime \prime}$ and since $\Gamma_{\mu, \rho, f}^{\prime \prime}$ itself is symmetric with respect to the $x=y$ line, it is clear $(-\alpha,-\beta) \epsilon$ $\Gamma_{\mu, \rho, f}^{\prime \prime}$ as desired. The proof is complete.

Let $\varphi=\left\{\varphi_{k}\right\}_{k \in \mathbf{Z}}$ be a solution of (5). Then $\varphi$ is uniquely determined by two consecutive terms $\varphi_{m}$ and $\varphi_{m+1}$. Although we can calculate the next term $\varphi_{m+2}$ directly, the conic sections allow us to easily "track" the movements of the points from $\left(\varphi_{m}, \varphi_{m+1}\right)$ to $\left(\varphi_{m+1}, \varphi_{m+2}\right)$. Indeed, by Theorem 8 , we see that $\mathcal{O}(\varphi) \subseteq \Gamma_{\mu, \rho, f}$, where $f=\Gamma_{\mu, \rho}\left(\varphi_{m}, \varphi_{m+1}\right)$. Then by means of this conic section, we may plot the corresponding orbit as follows.

Tracking Procedure. Input $(\alpha, \beta) \in \mathbf{R}^{2}$.

(1) Plot $\Gamma_{\mu, \rho, f}$ where $f=\Gamma_{\mu, \rho}(\alpha, \beta)$ on the plane.

(2) Take $k=0$ and start at the point $P_{k}=\left(x_{k}, y_{k}\right)=$ $(\alpha, \beta)$.

(3) Let $P_{k}^{\prime}=\left(y_{k}, x_{k}\right)$ be the point on $\Gamma_{\mu, \rho, f}$ which is symmetric to $P_{k}$ with respect to the $x=y$ line.

(4) Draw a vertical line $L_{k}$ through $P_{k}^{\prime}$.

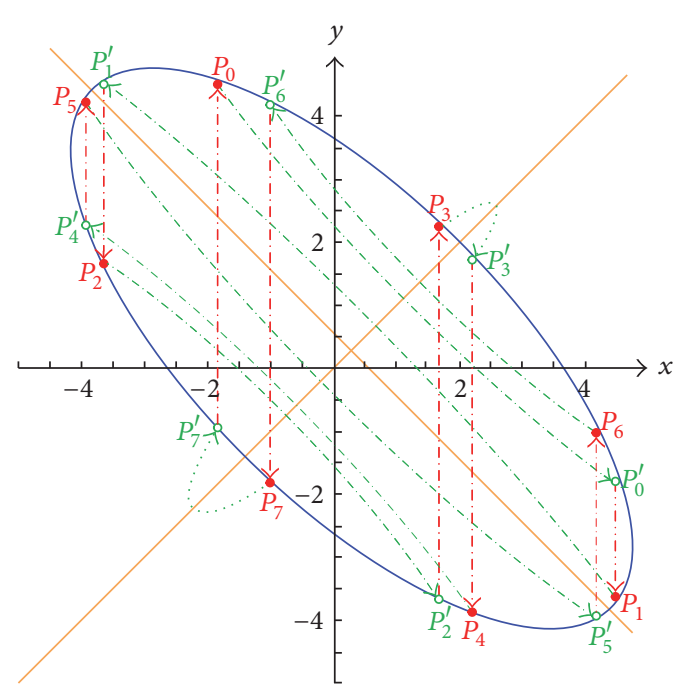

Figure 7: The orbit on $\bar{\Gamma}_{1, \sqrt{2}, 4,4 \sqrt{2}}$.

(5) The line $L_{k}$ and the conic section $\Gamma_{\mu, \rho, f}$ can intersect at $P_{k}^{\prime}$ and possibly at another point $P_{k}^{\perp}$. If $P_{k}^{\prime}=P_{k}^{\perp}$, then let $P_{k+1}=P_{k}^{\prime}$; otherwise, take $P_{k+1}=P_{k}^{\perp}$.

(6) If $P_{k+1}=P_{0}$, then stop; otherwise, let $P_{k}=P_{k+1}$ and go to step (3).

Note that by the Principal Axes Theorem, the level curve $\Gamma_{\mu, \rho, f}$ is defined by setting $\Gamma_{\mu, \rho}=f$, which is a polynomial of degree 2. The conic section has one principal on the $x=y$ line which implies any vertical line through $P_{k}^{\prime}$ meets $\Gamma_{\mu, \rho, f}$ at least once and at most twice through two points $P_{k}^{\prime}$ or $P_{k}^{\perp}$. If $P_{k}^{\prime}=P_{k}^{\perp}$, then $P_{k}^{\prime}$ is on the $\rho x+2 y=\mu$ line and this can be verified directly by (7). In view of the Tracking Procedure, we can generate each $P_{k}=\left(x_{k}, y_{k}\right)$ as $\left(\varphi_{k}, \varphi_{k+1}\right)$ by initializing $P_{0}=\left(x_{0}, y_{0}\right)=\left(\varphi_{0}, \varphi_{1}\right)$ and it is not difficult to see that the above algorithm can yield the "positive" orbit $\mathcal{O}^{+}(\varphi)$; on the other hand, the "negative" orbit of $\mathcal{O}(\varphi)$ can be plotted by the "positive" orbit $\mathcal{O}_{\perp}^{+}(\varphi)$ which is generated by the Tracking Procedure with input $\left(\varphi_{1}, \varphi_{0}\right)$. In Figures 7 and 8 , we illustrate the orbits of $P_{k}$ on ellipses; Figures 9 and 10 depict the orbits on a parabola and a degenerate parabola correspondingly; and in Figures 11 and 12, we show the orbits on a degenerate hyperbola and a hyperbola, respectively.

As we have shown previously, the $f$-level curve $\Gamma_{\mu, \rho, f}$ of $\Gamma_{\mu, \rho}$ is a conic section and it can be classified into hyperbola, ellipse, or parabola (or the degenerate curves). Let $\varphi=$ $\left\{\varphi_{k}\right\}_{k \in \mathbf{Z}}$ be a solution of (7) defined by $\left(\varphi_{0}, \varphi_{1}\right)=(\alpha, \beta)$. By means of the Tracking Procedure, we can easily analyze the asymptotic behavior of $\varphi_{k}$ when $k \rightarrow \infty$. In the sequel, we will discuss the sequence $\left\{\left(x_{k}, y_{k}\right)\right\}_{k \in \mathbf{N}}$ which is generated by the Tracking Procedure with input $\left(x_{0}, y_{0}\right)=(\alpha, \beta)$ depending on $|\rho|>2,|\rho|=2$, and $|\rho|<2$.

We first consider the case where $|\rho|>2$. By the previous discussions, the points $P_{k} \in\left\{\left(x_{k}, y_{k}\right)\right\}_{k \in \mathbf{N}}$ generated by the Tracking Procedure with input $\left(x_{0}, y_{0}\right)$ move on $\Gamma_{\mu, \rho, f}$ where $f=\Gamma_{\mu, \rho}\left(x_{0}, y_{0}\right)$ and $\Gamma_{\mu, \rho, f}$ is a hyperbola (or a degenerate one made up of two lines). Since $\Gamma_{\mu, \rho, f}$ is a hyperbola, we 


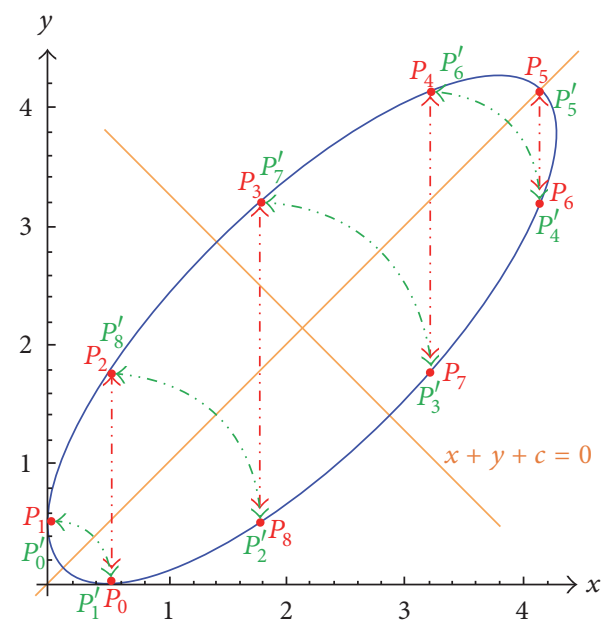

FIgURE 8: The orbit on $\bar{\Gamma}_{1,-2 \cos (2 \pi / 9),-1 / 4}$.

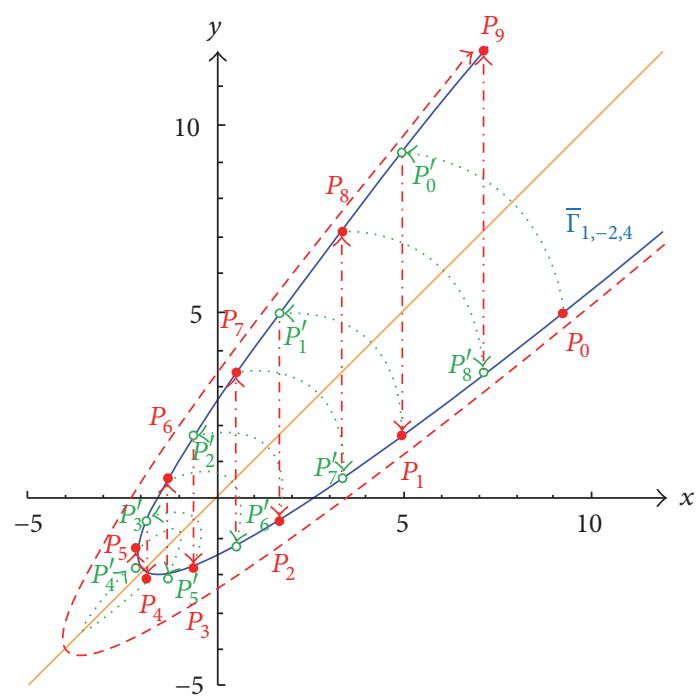

Figure 9: The orbit on $\bar{\Gamma}_{1,-2,4}$.

first locate the asymptotes of $\Gamma_{\mu, \rho, f}$. Note that $P_{c}=(\mu /(2+$ $\rho), \mu /(2+\rho))$ is the center of $\Gamma_{\mu, \rho, f}$. In view of $(21)$, we rewrite the equation $\Gamma_{\mu, \rho, f}$ as

$$
\begin{aligned}
y_{ \pm}(x)= & -\frac{1}{2}(\rho x-\mu) \\
& \pm \sqrt{\frac{1}{4}(\rho x-\mu)^{2}-x^{2}+\mu x+f},
\end{aligned}
$$

and suppose $y=m_{ \pm} x+n_{ \pm}$are the oblique asymptotes of $\Gamma_{\mu, \rho, f}$. Then we have

$$
\begin{aligned}
m_{ \pm} & =\lim _{x \rightarrow \infty^{+}} \frac{y_{ \pm}(x)}{x} \\
& =\lim _{x \rightarrow \infty^{+}}-\frac{1}{2}\left(\rho-\frac{\mu}{x}\right)
\end{aligned}
$$

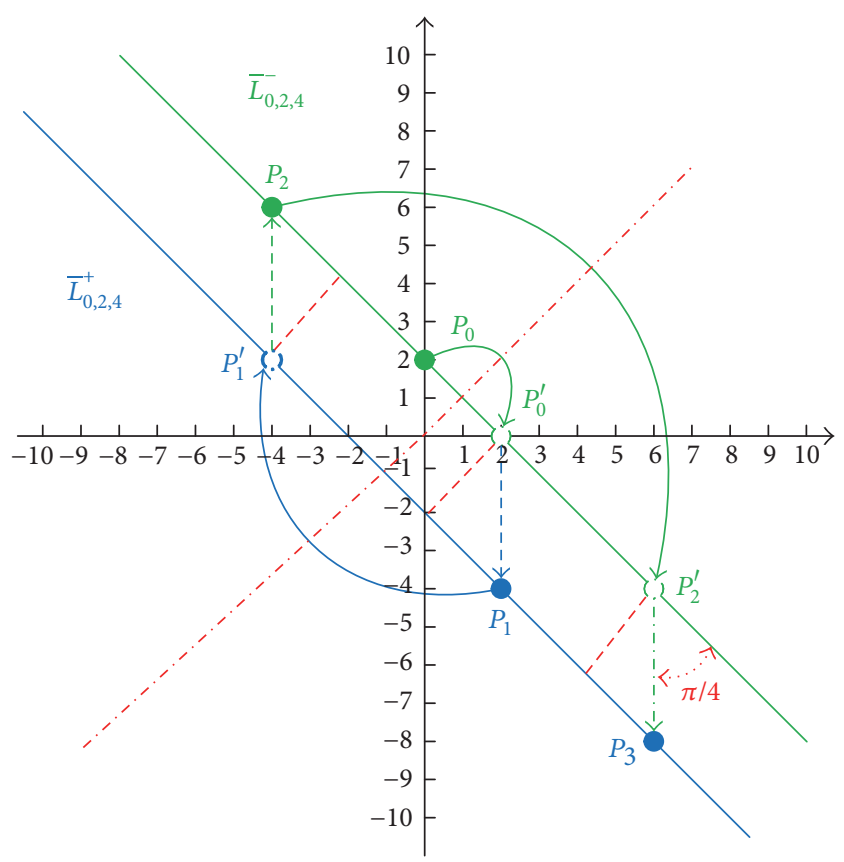

Figure 10: The orbit on $\bar{\Gamma}_{0,2,4}$.

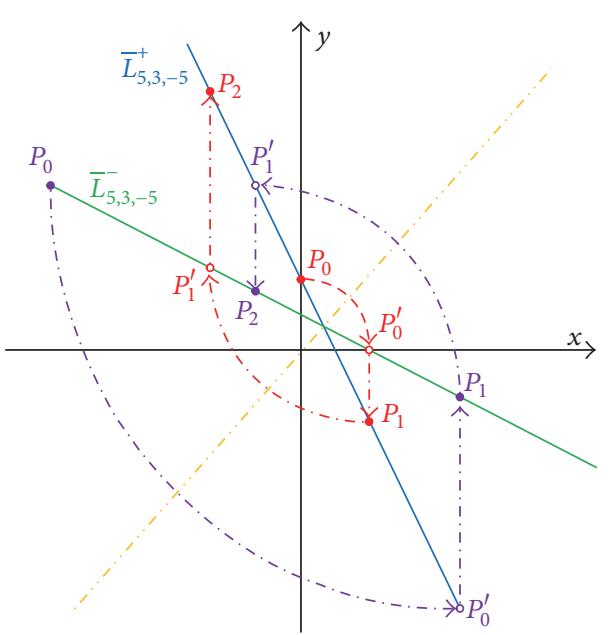

FIgURE 11: The orbit on $\bar{\Gamma}_{5,3,-5}$.

$$
\begin{aligned}
& \pm \sqrt{\frac{1}{4}\left(\rho-\frac{\mu}{x}\right)^{2}-1+\frac{\mu}{x}+\frac{f}{x^{2}}} \\
= & -\frac{1}{2} \rho \pm \frac{1}{2} \sqrt{\rho^{2}-4} .
\end{aligned}
$$

As for $n_{ \pm}$, since lines $y=m_{ \pm} x+n_{ \pm}$intersect at $P_{c}$, we apply $P_{c}$ to the lines and obtain $n_{ \pm}=\left(\mu-\mu m_{ \pm}\right) /(2+\rho)$. Accordingly, we see that $\bar{L}_{\mu, \rho, f}^{+}$and $\bar{L}_{\mu, \rho, f}^{-}$can be expressed by $y-m_{-} x-n_{-}=0$ as well as $y-m_{+} x-n_{+}=0$, respectively. For $|\rho|>2$, one 


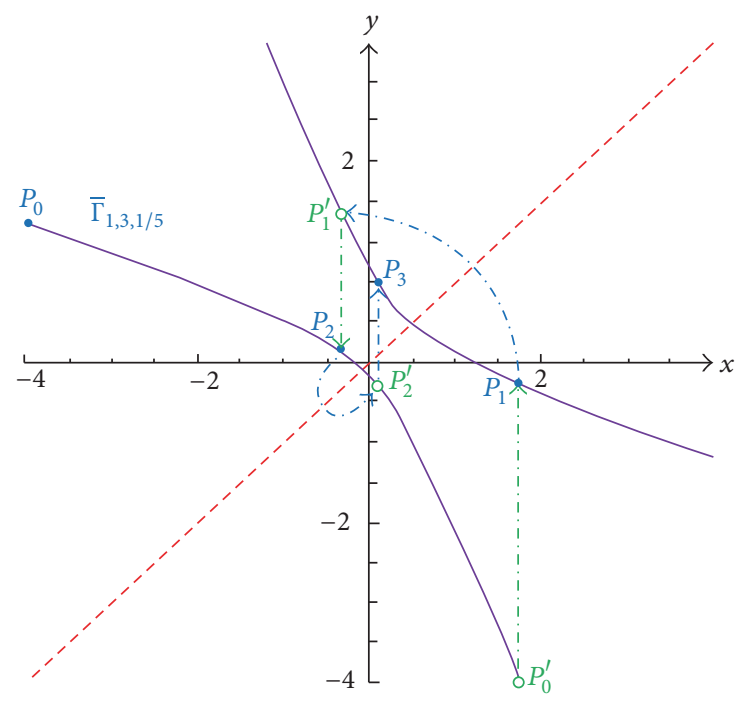

FIgURE 12: The orbit on $\bar{\Gamma}_{1,3,1 / 5}$.

relation between $P_{k}$ and $L_{\mu, \rho, f}^{+}$as well as $L_{\mu, \rho, f}^{-}$defined in (24) and (25), respectively, is revealed by

$$
L_{\mu, \rho, f}^{+}\left(y_{k}, \mu-\rho y_{k}-x_{k}\right)=-L_{\mu, \rho, f}^{-}\left(y_{k}, x_{k}\right)
$$

as well as

$$
L_{\mu, \rho, f}^{-}\left(y_{k}, \mu-\rho y_{k}-x_{k}\right)=-L_{\mu, \rho, f}^{+}\left(y_{k}, x_{k}\right) .
$$

Moreover, with the oblique asymptotes $\bar{L}_{\mu, \rho, f}^{+}$and $\bar{L}_{\mu, \rho, f}^{-}$, we may illustrate the asymptotic behaviors of $\Gamma_{\mu, \rho, f}$ where $|\rho|>2$.

Theorem 9. Suppose $|\rho|>2$ and let $(\alpha, \beta) \in \mathbf{R}^{2}$ such that $f=\Gamma_{\mu, \rho}(\alpha, \beta)$. Let $\left\{\left(x_{k}, y_{k}\right)\right\}_{k \in \mathbf{N}}$ be generated by the Tracking Procedure with input $\left(x_{0}, y_{0}\right)=(\alpha, \beta)$. Then $\Gamma_{\mu, \rho, f}$ is a hyperbola (or a degenerate one made up of two lines $\bar{L}_{\mu, \rho, f}^{-}$ and $\bar{L}_{\mu, \rho, f}^{+}$) and we have the following.

(i) Suppose $\Gamma_{\mu, \rho, f}$ is a degenerate hyperbola. If $(\alpha, \beta) \in$ $\bar{L}_{\mu, \rho, f}^{+}$, then $\left(x_{m}, y_{m}\right) \in \bar{L}_{\mu, \rho, f}^{+}$for all $m \in \mathbf{N}$; if $(\alpha, \beta) \epsilon$ $\bar{L}_{\mu, \rho, f}^{-}$, then $\left(x_{n}, y_{n}\right) \in \bar{L}_{\mu, \rho, f}^{-}$for all $n \in \mathbf{N}$.

(ii) Suppose $\Gamma_{\mu, \rho, f}$ is a hyperbola. If $\rho \geq 0$, then $\left(x_{m}, x_{m+1}\right)$ and $\left(x_{m+1}, x_{m+2}\right)$ are sitting on different branches for all $m \in \mathbf{N}$; if $\rho<0$, then $\left(x_{n}, x_{n+1}\right)$ and $\left(x_{n+1}, x_{n+2}\right)$ lie on the same branch for all $n \in \mathbf{N}$.

Proof. Note that $x_{0}=\alpha$ and $x_{1}=y_{0}=\beta$. By Theorem 8 and (7), we see that $y_{1}=\mu-\rho y_{0}-x_{0}=\mu-\rho \beta-\alpha=$ $\gamma$. Suppose $(\alpha, \beta) \in \bar{L}_{\mu, \rho, f}^{+}$or $(\alpha, \beta) \in \bar{L}_{\mu, \rho, f}^{-}$. In the former case, $\bar{L}_{\mu, \rho, f}^{+}(\alpha, \beta)=\bar{L}_{\mu, \rho, f}^{-}(\beta, \alpha)=0$ and by (31), as well as (32), $\bar{L}_{\mu, \rho, f}^{+}(\beta, \gamma)=-\bar{L}_{\mu, \rho, f}^{-}(\beta, \alpha)=0$ which implies $\left(x_{1}, y_{1}\right)=$ $(\beta, \gamma) \in \bar{L}_{\mu, \rho, f}^{+}$; in the latter case, by similar arguments, we may see that $\bar{L}_{\mu, \rho, f}^{-}(\beta, \gamma)=0$. Note that if $(\alpha, \beta) \in \bar{L}_{\mu, \rho, f}^{+} \cap$ $\bar{L}_{\mu, \rho, f}^{-}$, then $(\alpha, \beta)=(\mu /(2+\rho), \mu /(2+\rho))$ and by the Tracking
Procedure, $\left(x_{1}, y_{1}\right)=(\mu /(2+\rho), \mu /(2+\rho))$ which implies $\left(x_{i}, y_{i}\right)=(\mu /(2+\rho), \mu /(2+\rho))$ for all $i \in \mathbf{N}$. Suppose $(\alpha, \beta) \notin$ $\bar{L}_{\mu, \rho, f}^{+} \cup \bar{L}_{\mu, \rho, f}^{-}$. Then $\Gamma_{\mu, \rho, f}$ is a hyperbola and $(\alpha, \beta)$ is on one branch of $\Gamma_{\mu, \rho, f}$. By the Tracking Procedure, we may see that if $\rho \geq 0$, then $P_{1}$ "jumps" to another branch (see Figure 3 ), while if $\rho<0$, then $P_{1}$ will stay on the same branch (see Figure 4). By induction, we may see that (ii) holds. This completes our proof.

In view of Theorem 9, we may follow the movements of $\left(x_{k}, y_{k}\right)$ generated by the Tracking Procedure when $|\rho|>2$. This enables us to see the asymptotic behavior of $\left(x_{k}, y_{k}\right)$ when $k \rightarrow \infty$ in Theorem 10. Before introducing Theorem 10, we denote the distance between two points $P_{1}$ and $P_{2}$ on $\mathbf{R}^{2}$ by $\left|P_{1}-P_{2}\right|$ and the distance from a point $P$ to the line $L$ by $d(P, L)$.

Theorem 10. Suppose $|\rho|>2$ and let $(\alpha, \beta) \in \mathbf{R}^{2}$ such that $f=\Gamma_{\mu, \rho}(\alpha, \beta)$. Let $\left\{\left(x_{k}, y_{k}\right)\right\}_{k \in \mathbf{N}}$ be generated by the Tracking Procedure with $\left(x_{0}, y_{0}\right)=(\alpha, \beta)$. Then $\Gamma_{\mu, \rho, f}$ is a hyperbola (or a degenerate one made up of two lines $\bar{L}_{\mu, \rho, f}^{+}$and $\bar{L}_{\mu, \rho, f}^{-}$) and we have the following.

(i) Suppose $(\alpha, \beta) \in \bar{L}_{\mu, \rho, f}^{+} \cup \bar{L}_{\mu, \rho, f}^{-}$. If $(\alpha, \beta)=(\mu /(2+$ $\rho), \mu /(2+\rho))$, then $\left(x_{k}, y_{k}\right)=(\alpha, \beta)$ for all $k \in \mathbf{N}$; if $(\alpha, \beta) \in \bar{L}_{\mu, \rho, f}^{+}$and $(\alpha, \beta) \neq(\mu /(2+\rho), \mu /(2+\rho))$, then

$$
\lim _{k \rightarrow \infty} d\left(\left(x_{k}, y_{k}\right), \bar{L}_{\mu, \rho, f}^{-}\right)= \begin{cases}\infty & \text { if } \rho>2 \\ 0 & \text { if } \rho<-2 ;\end{cases}
$$

and if $(\alpha, \beta) \in \bar{L}_{\mu, \rho, f}^{-}$and $(\alpha, \beta) \neq(\mu /(2+\rho), \mu /(2+\rho))$, then

$$
\lim _{k \rightarrow \infty} d\left(\left(x_{k}, y_{k}\right), \bar{L}_{\mu, \rho, f}^{+}\right)= \begin{cases}0 & \text { if } \rho>2 \\ \infty & \text { if } \rho<-2 .\end{cases}
$$

(ii) If $\rho>2$ and $\Gamma_{\mu, \rho, f}$ is a hyperbola, then $\lim _{k \rightarrow \infty} d\left(\left(x_{k}\right.\right.$, $\left.\left.y_{k}\right), \bar{L}_{\mu, \rho, f}^{+}\right)=0$; and if $\rho<-2$ and $\Gamma_{\mu, \rho, f}$ is a hyperbola, then $\lim _{k \rightarrow \infty} d\left(\left(x_{k}, y_{k}\right), \bar{L}_{\mu, \rho, f}^{-}\right)=0$.

Proof. For the sake of convenience, we let $P_{c}=(\mu /(2+$ $\rho), \mu /(2+\rho))$ and $P_{k}=\left(x_{k}, y_{k}\right)$ so that $P_{0}=(\alpha, \beta)$ and $f=\Gamma_{\mu, \rho}(\alpha, \beta)$. Let $y_{1}=\gamma=\mu-\rho \beta-\alpha$. By Proposition 2, if $P_{0}=P_{c}$, then $\varphi$ is a constant solution of (7) which implies $\left\{P_{k}\right\}_{k \in \mathbf{N}}=\left\{P_{c}\right\}$. Now, we suppose $P_{0} \neq P_{c}$. Since $|\rho|>2$, $\Gamma_{\mu, \rho, f}$ is a hyperbola (or a degenerate one). Hence, we discuss the two cases where $P_{0}$ is on a degenerate hyperbola or a hyperbola.

Suppose $P_{0} \in \bar{L}_{\mu, \rho, f}^{+} \cup \bar{L}_{\mu, \rho, f}^{-}$and $P_{0} \neq P_{c}$. Then by Theorem $9, P_{0}^{\prime}$ and $P_{1}^{\prime}$ are on the same line. In view of (24) and (25), we let $m_{+}=\rho+\delta, m_{-}=\rho-\delta, \Delta_{+}=\mu+\delta /(2+\rho)$, and $\Delta_{+}=\mu-\delta /(2+\rho)$. For the sake of convenience, we also let

$$
\kappa=\sqrt{\frac{1+m_{-}^{2}}{1+m_{+}^{2}}} .
$$


Since $\Gamma_{\mu, \rho, f}$ is symmetric with respect to the $x=y$ line, we see that if $\rho>2$, then $\kappa<1$; if $\rho<-2$, then $\kappa>1$. Suppose $\rho>2$. If $P_{0} \in \bar{L}_{\mu, \rho, f}^{-}$and $P_{0} \neq P_{c}$, then $P_{0}^{\prime} \in \bar{L}_{\mu, \rho, f}^{+}$and $P_{1} \in \bar{L}_{\mu, \rho, f}^{-}$so that $\alpha+m_{+} \beta+\Delta_{+}=0$ and $\gamma+m_{-} \beta+\Delta_{-}=0$, respectively. Also, by the symmetry of $\Gamma_{\mu, \rho, f}$ with respect to the $x=y$ line, we can be sure that $d\left(P_{0}, \bar{L}_{\mu, \rho, f}^{+}\right)=d\left(P_{0}^{\prime}, \bar{L}_{\mu, \rho, f}^{-}\right)$so that

$$
d\left(P_{0}, \bar{L}_{\mu, \rho, f}^{+}\right)=\frac{\left|\alpha+m_{-} \beta+\Delta_{-}\right|}{\sqrt{1+m_{-}^{2}}}=\frac{|\alpha-\gamma|}{\sqrt{1+m_{-}^{2}}} .
$$

On the other hand, we calculate

$$
d\left(P_{1}, \bar{L}_{\mu, \rho, f}^{+}\right)=\frac{\left|\gamma+m_{+} \beta+\Delta_{+}\right|}{\sqrt{1+m_{+}^{2}}}=\frac{|\gamma-\alpha|}{\sqrt{1+m_{+}^{2}}}
$$

and by (35) and (36), as well as (37), it follows that

$$
d\left(P_{1}, \bar{L}_{\mu, \rho, f}^{+}\right)=\kappa d\left(P_{0}, \bar{L}_{\mu, \rho, f}^{+}\right) .
$$

By induction, we have $d\left(P_{i}, \bar{L}_{\mu, \rho, f}^{+}\right)=\kappa^{i} d\left(P_{0}, \bar{L}_{\mu, \rho, f}^{+}\right)$, where $\kappa<1$ and $i \in \mathbf{N}$. Accordingly, (34) holds. The case where $\rho<$ -2 and $P_{0} \in \bar{L}_{\mu, \rho, f}^{-} \backslash\left\{P_{c}\right\}$ can be handled similarly with $d\left(P_{j}\right.$, $\left.\bar{L}_{\mu, \rho, f}^{+}\right)=\kappa^{-j} d\left(P_{0}, \bar{L}_{\mu, \rho, f}^{+}\right)$, where $\kappa>1$ and $j \in \mathbf{N}$. By similar arguments, (33) is also true.

Suppose $P_{0} \notin \bar{L}_{\mu, \rho, f}^{+} \cup \bar{L}_{\mu, \rho, f}^{-}$. We first consider the case where $\rho>2$. Then $\kappa<1$ and by (24)

$$
\begin{aligned}
d\left(P_{1}, \bar{L}_{\mu, \rho, f}^{+}\right) & =\frac{\left|L_{\mu, \rho, f}^{+}(\beta, \gamma)\right|}{\sqrt{1+m_{+}^{2}}}=\frac{\left|-L_{\mu, \rho, f}^{-}(\beta, \alpha)\right|}{\sqrt{1+m_{+}^{2}}} \\
& =\frac{\left|-L_{\mu, \rho, f}^{-}(\beta, \alpha)\right|}{\sqrt{1+m_{-}^{2}}} \frac{\sqrt{1+m_{-}^{2}}}{\sqrt{1+m_{+}^{2}}} \\
& =\kappa d\left(P_{0}^{\prime}, \bar{L}_{\mu, \rho, f}^{-}\right)=\kappa d\left(P_{0}, \bar{L}_{\mu, \rho, f}^{+}\right) .
\end{aligned}
$$

By induction, it follows that $d\left(P_{i}, \bar{L}_{\mu, \rho, f}^{+}\right)=\kappa^{i} d\left(P_{0}, \bar{L}_{\mu, \rho, f}^{+}\right)$, where $i \in \mathbf{N}$. Consequently, case (ii) is true when $\rho>2$ and by similar arguments, we also see that $\rho<-2$ holds with $d\left(P_{j}, \bar{L}_{\mu, \rho, f}^{-}\right)=\kappa^{-j} d\left(P_{0}, \bar{L}_{\mu, \rho, f}^{-}\right)$, where $j \in \mathbf{N}$ and $\kappa>1$.

The proof is complete.

Corollary 11. Suppose $|\rho|>2$ and let $(\alpha, \beta) \in \mathbf{R}^{2}$ such that $f=\Gamma_{\mu, \rho}(\alpha, \beta)$. Let $\left\{\left(x_{k}, y_{k}\right)\right\}_{k \in \mathbf{N}}$ be generated by the Tracking Procedure with input $\left(x_{0}, y_{0}\right)=(\alpha, \beta)$. Then $\Gamma_{\mu, \rho, f}$ is a hyperbola (or a degenerate one made up of two lines $\bar{L}_{\mu, \rho, f}^{+}$ and $\left.\bar{L}_{\mu, \rho, f}^{-}\right)$. Suppose $(\alpha, \beta) \neq(\mu /(2+\rho), \mu /(2+\rho))$. If $d((\alpha, \beta)$, $\left.\bar{L}_{\mu, \rho, f}^{+}\right) \leq d\left((\alpha, \beta), \bar{L}_{\mu, \rho, f}^{-}\right)$, where $\rho>2$, then $x_{k} x_{k+1}<0$ and $\left|x_{k}\right| \rightarrow \infty$ when $k \rightarrow \infty$; if $d\left((\alpha, \beta), \bar{L}_{\mu, \rho, f}^{-}\right) \leq d\left((\alpha, \beta), \bar{L}_{\mu, \rho, f}^{+}\right)$, where $\rho<-2$, then $x_{k} x_{k+1}>0$ and $\left|x_{k}\right| \rightarrow \infty$ when $k \rightarrow \infty$.

The argument of Corollary 11 can be verified directly from Theorem 10 based on the facts that the slope of $\bar{L}_{\mu, \rho, f}^{-}$

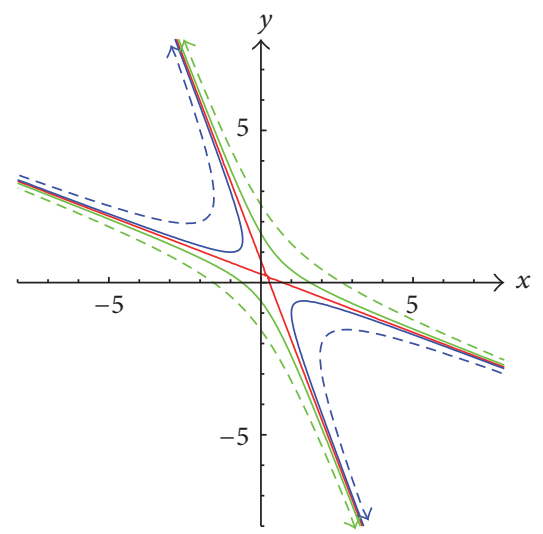

FIGURE 13: The asymptotic behavior of $\left\{P_{k}\right\}$ plotted by Tracking Procedure with $\rho>2$.

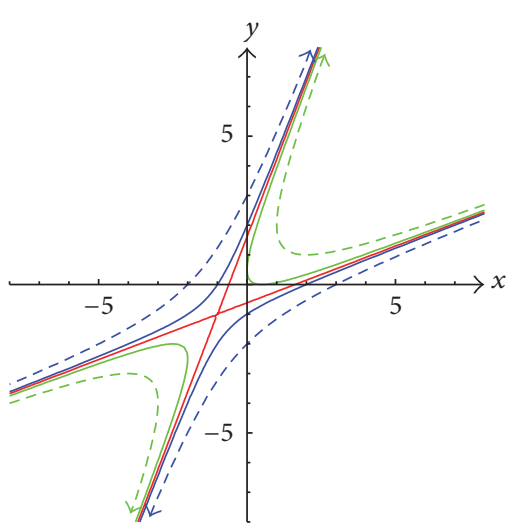

FIgURE 14: The asymptotic behavior of $\left\{P_{k}\right\}$ plotted by Tracking Procedure with $\rho<-2$.

is characterized by $-\rho+\delta<0$ and the slope of $\bar{L}_{\mu, \rho, f}^{+}$is characterized by $-\rho-\delta>0$, where $\rho>2$ and $\rho<-2$, respectively, and, thus, the proof is omitted.

In Theorem 10, we showed the asymptotic behaviors of $\left\{P_{k}\right\}_{k \in \mathbf{N}}$ generated by the Tracking Procedure. We illustrate the movements of $\left\{P_{k}\right\}_{k \in \mathbf{N}}$ when $k \rightarrow \infty$ in Figures 13, 14, and 15 where the arrows indicate the moving directions of $P_{k}$ as $k \rightarrow \infty$.

Next, we consider the asymptotic behaviors of $\left\{P_{k}\right\}_{k \in \mathbf{N}}$ with $\rho=2$ or $\rho=-2$.

Theorem 12. Suppose $\rho=2$ and let $(\alpha, \beta) \in \mathbf{R}^{2}$ such that $f=$ $\Gamma_{\mu, 2}(\alpha, \beta)$. Let $\left\{\left(x_{k}, y_{k}\right)\right\}_{k \in \mathbf{N}}$ be generated by the Tracking Procedure with input $\left(x_{0}, y_{0}\right)=(\alpha, \beta)$. Then $f \geq-\mu^{2} / 4$ and $\Gamma_{\mu, \rho, f}$ is a degenerate parabola made up of $\bar{L}_{\mu, 2,-\mu^{2} / 4}^{+}$and $\bar{L}_{\mu, 2,-\mu^{2} / 4}^{-}$. If $f=-\mu^{2} / 4$, then $\bar{L}_{\mu, 2,-\mu^{2} / 4}^{+}=\bar{L}_{\mu, 2,-\mu^{2} / 4}^{-}$and $\left(x_{k}, y_{k}\right)=\left(x_{k+2}\right.$, $\left.y_{k+2}\right)$ for all $k \in \mathbf{N}$; otherwise, $\left(x_{k}, y_{k}\right)$ as well as $\left(x_{k+1}, y_{k+1}\right)$ are not on the same line. Furthermore, if $f>-\mu^{2} / 4$, then $d\left(\left(x_{k}, y_{k}\right), \bar{\Pi}\right) \rightarrow \infty$ and $x_{k} y_{k}<0$ when $k \rightarrow \infty$. 
Proof. For the sake of convenience, we let $P_{c}=(\mu /(2+$ $\rho), \mu /(2+\rho))$ and $P_{k}=\left(x_{k}, y_{k}\right)$. First of all, we see that

$$
\begin{aligned}
\Gamma_{\mu, 2}(x, y) & =x^{2}+2 x y+y^{2}-\mu x-\mu y \\
& =(x+y)^{2}-\mu(x+y) \\
& =\left(x+y-\frac{\mu}{2}\right)^{2}-\frac{1}{4} \mu^{2},
\end{aligned}
$$

which implies $f \geq-\mu^{2} / 4$. Furthermore,

$$
\begin{gathered}
\Gamma_{\mu, 2, f}=\left(x+y-\frac{1}{2} \mu-\sqrt{f+\frac{1}{4} \mu^{2}}\right) \\
\cdot\left(x+y-\frac{1}{2} \mu+\sqrt{f+\frac{1}{4} \mu^{2}}\right)=0,
\end{gathered}
$$

which leads to $\Gamma_{\mu, 2, f}$ being two parallel lines. Consequently, if $f=-\mu^{2} / 4$, then $\bar{L}_{\mu, 2,-\mu^{2} / 4}^{+}=\bar{L}_{\mu, 2,-\mu^{2} / 4}^{-}$; otherwise, $\bar{L}_{\mu, 2,-\mu^{2} / 4}^{+}$ and $\bar{L}_{\mu, 2,-\mu^{2} / 4}^{-}$are two distinct parallel lines. Suppose $f=$ $-\mu^{2} / 4$. Then $P_{0} \in \bar{L}_{\mu, 2,-\mu^{2} / 4}^{+}=\bar{L}_{\mu, 2,-\mu^{2} / 4}^{-}$. In view of the Tracking Procedure, it is found that if $P_{0}=P_{c}$, then $P_{1}=P_{0}$ which implies $P_{m}=P_{0}$ for all $m \in \mathbf{N}$; otherwise, $P_{1}^{\prime}=$ $P_{1}^{\perp}=P_{2}=P_{0}$ which leads to $P_{n}=P_{n+2}$ for all $n \in \mathbf{N}$. Next, suppose $f>-\mu^{2} / 4$. By the Tracking Procedure, we can directly observe (see Figure 10) that for an arbitrary $n \in \mathbf{N}$, if $\left(x_{n}, y_{n}\right) \in \bar{L}_{\mu, 2, f}^{+}$, then $\left(x_{n+1}, y_{n+1}\right) \in \bar{L}_{\mu, 2, f}^{-}$and vice versa. Now, we discuss the asymptotic behaviors of $\left\{\left(x_{k}, y_{k}\right)\right\}_{k \in \mathbf{N}}$. By the Tracking Procedure, we first observe that

$$
\begin{aligned}
& d\left(P_{m+1}, \Pi\right) \\
& = \begin{cases}\left(P_{m}, \bar{\Pi}\right)+d\left(\bar{L}_{\mu, 2, f}^{+}, \bar{L}_{\mu, 2, f}^{-}\right) & \text {if } d\left(P_{m+1}, \bar{\Pi}\right)>d\left(P_{m}, \bar{\Pi}\right), \\
\left(P_{m}, \bar{\Pi}\right)-d\left(\bar{L}_{\mu, 2, f}^{+}, \bar{L}_{\mu, 2, f}^{-}\right) & \text {if } d\left(P_{m+1}, \bar{\Pi}\right)<d\left(P_{m}, \bar{\Pi}\right),\end{cases}
\end{aligned}
$$

and clearly, the converses are also true. Note that $d\left(P_{n}, \Pi\right) \neq$ $d\left(P_{n+1}, \Pi\right)$ for any $n \in \mathbf{N}$. Furthermore, by the Tracking Procedure again, if $d\left(P_{1}, \bar{\Pi}\right)>d\left(P_{0}, \bar{\Pi}\right)$, then for all $k \in \mathbf{N}$

$$
d\left(P_{k}, \bar{\Pi}\right)=d\left(P_{0}, \bar{\Pi}\right)+k d\left(\bar{L}_{\mu, 2, f}^{+}, \bar{L}_{\mu, 2, f}^{-}\right) ;
$$

otherwise, we see that for some $j \in \mathbf{N}$,

$$
d\left(P_{j}, \bar{\Pi}\right)=d\left(P_{0}, \bar{\Pi}\right)-j d\left(\bar{L}_{\mu, 2, f}^{+}, \bar{L}_{\mu, 2, f}^{-}\right) .
$$

In view of (44), since $d\left(P_{j}, \bar{\Pi}\right) \geq 0, d\left(P_{0}, \bar{\Pi}\right) \geq 0$, and $d\left(\bar{L}_{\mu, 2, f}^{+}, \bar{L}_{\mu, 2, f}^{-}\right)$is a positive constant, there is $i \in \mathbf{N}$ such that $d\left(P_{i}, \bar{\Pi}\right) \geq 0$ as well as $d\left(P_{i+1}, \bar{\Pi}\right)<0$ and, thus, by the previous discussions, $d\left(P_{i+1}, \bar{\Pi}\right)>d\left(P_{i}, \bar{\Pi}\right)$. Accordingly, by the previous discussions again, $d\left(P_{k}, \bar{\Pi}\right) \rightarrow \infty$ when $k \rightarrow \infty$ and since the slopes of $\bar{L}_{\mu, 2, f}^{+}$and $\bar{L}_{\mu, 2, f}^{-}$are $-1, x_{k} y_{k}<0$ as $k \rightarrow \infty$. The proof is complete.

Next, we consider the case where $\rho=-2$.
Theorem 13. Suppose $\rho=-2$ and let $(\alpha, \beta) \in \mathbf{R}^{2}$ such that $f=\Gamma_{\mu,-2}(\alpha, \beta)$. Let $\left\{\left(x_{k}, y_{k}\right)\right\}_{k \in \mathbf{N}}$ be generated by the Tracking Procedure with input $\left(x_{0}, y_{0}\right)=(\alpha, \beta)$. Then we have the following.

(i) Suppose $\mu=0$. Then $f \geq 0$ and $\Gamma_{0,-2, f}$ is a degenerate parabola made up of two parallel lines $\bar{L}_{0,-2, f}^{+}$and $\bar{L}_{0,-2, f}^{-}$. If $f=0$, then $\bar{L}_{0,-2, f}^{+}=\bar{L}_{0,-2, f}^{-}=\bar{\Pi}$ and $\alpha=$ $\beta$ such that $\left(x_{k}, y_{k}\right)=(\alpha, \alpha)$ for all $k \in \mathbf{N}$; otherwise, $\left(x_{k}, y_{k}\right)$ and $\left(x_{k+1}, y_{k+1}\right)$ are on the same line and $d\left(\left(x_{k}, y_{k}\right), P_{0}\right) \rightarrow \infty$ when $k \rightarrow \infty$.

(ii) Suppose $\mu \neq 0$. Then $\Gamma_{\mu,-2, f}$ is a parabola and $d\left(\left(x_{k}\right.\right.$, $\left.\left.y_{k}\right), \bar{\Pi}\right) \rightarrow \infty$ when $k \rightarrow \infty$.

Proof. Suppose $\mu=0$. By (20), $\Gamma_{0,-2}(x, y)=x^{2}-2 x y+$ $y^{2}=(x-y)^{2}$ and, thus, $f=\Gamma_{0,-2}(\alpha, \beta)=(\alpha-\beta)^{2} \geq 0$. Accordingly, $\Gamma_{0,-2, f}$ is a degenerate parabola which is made up of two parallel lines $\bar{L}_{0,2, f}^{+}$and $\bar{L}_{0,2, f}^{-}$as defined in (24) and (25), respectively. More precisely, if $\alpha=\beta$, then $f=0$ which leads to $\bar{L}_{0,2,0}^{+}=\bar{L}_{0,2,0}^{-}=\bar{\Pi}$; if $\alpha \neq \beta$, then $f>0$ which implies $\bar{L}_{0,2, f}^{+}$and $\bar{L}_{0,2, f}^{-}$are two distinct parallel lines. By the Tracking Procedure, if $\alpha=\beta$, then $\left(x_{k}, y_{k}\right)=(\alpha, \alpha)$ for all $k \in \mathbf{N}$; if $\alpha \neq \beta$, then $\left(x_{k}, y_{k}\right)$ and $\left(x_{k+1}, y_{k+1}\right)$ are on the same line. Furthermore, if $\alpha \neq \beta$, then we have $\Delta=d\left(\bar{L}_{0,-2, f}^{+}, \bar{L}_{0,-2, f}^{-}\right)=\sqrt{2 f}$ and by the Tracking Procedure again, $d\left(\left(x_{k}, y_{k}\right),(\alpha, \beta)\right)=k \Delta \tan (\pi / 4)=k \Delta$, where $k \in \mathbf{N}$. Accordingly, $d\left(\left(x_{k}, y_{k}\right), P_{0}\right) \rightarrow \infty$ when $k \rightarrow \infty$. Next, suppose $\mu \neq 0$. Then $\Gamma_{\mu,-2, f}$ is parabola. By (7), for $k \in \mathbf{N}$, we have $y_{k}=x_{k+1}$ such that $x_{k+2}-x_{k+1}=\mu+x_{k+1}-x_{k}$ and thus

$$
\begin{aligned}
d\left(\left(x_{k}, y_{k}\right), \bar{\Pi}\right) & =\frac{\left|\mu+2 x_{k+1}-x_{k}-x_{k+1}\right|}{\sqrt{2}} \\
& =\frac{\left|\mu+x_{k+1}-x_{k}\right|}{\sqrt{2}}
\end{aligned}
$$

which leads to

$$
d\left(\left(x_{k}, y_{k}\right), \bar{\Pi}\right)=\frac{1}{\sqrt{2}}|k \mu+(\beta-\alpha)|
$$

Hence, $d\left(\left(x_{k}, y_{k}\right), \bar{\Pi}\right) \rightarrow \infty$ when $k \rightarrow \infty$. Note that, if $\mu>0$, then $\Gamma_{\mu,-2, f}$ is concave upward (see Figure 15), while if $\mu<0$, then $\Gamma_{\mu,-2, f}$ is concave downward. Accordingly, (ii) is true and this completes the proof.

Corollary 14. Suppose $\rho=-2$ and let $(\alpha, \beta) \in \mathbf{R}^{2}$ such that $f=\Gamma_{\mu,-2}(\alpha, \beta)$. Let $\left\{\left(x_{k}, y_{k}\right)\right\}_{k \in \mathbf{N}}$ be generated by the Tracking Procedure with input $\left(x_{0}, y_{0}\right)=(\alpha, \beta)$. Then we have the following.

(i) Suppose $\mu=0$. Then $f \geq 0$ and $\Gamma_{0,-2, f}$ is a degenerate parabola with two parallel lines, $\bar{L}_{0,-2, f}^{+}$and $\bar{L}_{0,-2, f}^{-}$. If $\alpha=\beta$, then $f=0$ such that $\bar{L}_{0,-2, f}^{+}=\bar{L}_{0,-2, f}^{-}=\bar{\Pi}$ and 


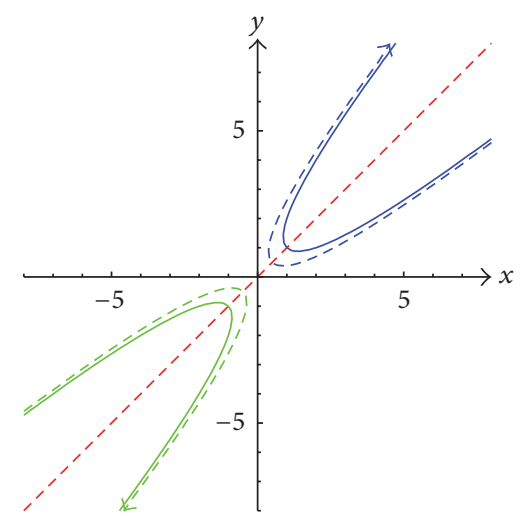

FIGURE 15: The asymptotic behavior of $\left\{P_{k}\right\}$ plotted by Tracking Procedure with $\rho=-2$.

$x_{k}=\alpha$ for all $k \in \mathbf{N}$; otherwise, $f>0$ such that $\bar{L}_{0,-2, f}^{+}$ as well as $\bar{L}_{0,-2, f}^{-}$are two distinct parallel lines and

$$
x_{k} \longrightarrow \begin{cases}\infty & \text { if }(\alpha, \beta) \in \bar{L}_{0,-2, f}^{+} \\ -\infty & \text { if }(\alpha, \beta) \in \bar{L}_{0,-2, f}^{-}\end{cases}
$$

(ii) Suppose $\mu \neq 0$. Then $\Gamma_{\mu,-2, f}$ is a parabola. If $\mu>0$, then $\Gamma_{\mu,-2, f}$ is concave upward and $x_{k} \rightarrow \infty$ when $k \rightarrow \infty$; otherwise, $\Gamma_{\mu,-2, f}$ is concave downward and $x_{k} \rightarrow-\infty$ when $k \rightarrow \infty$.

The results stated in Corollary 14 can be derived directly from Theorem 13 by observing the asymptotic behavior of the points $\left(x_{k}, y_{k}\right)$.

Let $\left\{\left(x_{k}, y_{k}\right)\right\}_{k \in \mathbf{N}}$ be generated by the Tracking Procedure with inputs $\left(x_{0}, y_{0}\right)=(\alpha, \beta)$ and $f=\Gamma_{\mu, \rho}(\alpha, \beta)$. In Theorems 10,12 , and 13, we have shown the asymptotic behaviors of $\varphi$ with $|\rho| \geq 2$. Next, we consider the case where $|\rho|<2$ and $(\alpha, \beta) \neq(\mu /(2+\rho), \mu /(2+\rho))$. Suppose $|\rho|<2$. Then the conic section $\Gamma_{\mu, \rho, f}$ is an ellipse which implies $x_{k}$ is both bounded above and below. Moreover, by the Principal Axes Theorem, the nondegenerate $\Gamma_{\mu, \rho, f}$ is an ellipse which is rotated by $\vartheta \in$ $\{-\pi / 4, \pi / 4\}$ and translated by $\left(x_{c}, y_{c}\right)=(\mu /(2+\rho), \mu /(2+$ $\rho)$ ) from a standard ellipse with major axis $a$ as well as minor axis $b$. Thus, $\Gamma_{\mu, \rho, f}$ where $f \neq-\mu^{2} /(2+\rho)^{2}$ can be expressed parametrically by

$$
\left[\begin{array}{l}
x(\theta) \\
y(\theta)
\end{array}\right]=\left[\begin{array}{l}
x_{c} \\
y_{c}
\end{array}\right]+\left[\begin{array}{cc}
\cos \vartheta & -\sin \vartheta \\
\sin \vartheta & \cos \vartheta
\end{array}\right]\left[\begin{array}{l}
a \cos \theta \\
b \sin \theta
\end{array}\right],
$$

where the polar angle $\theta \in[0,2 \pi]$. For the sake of convenience, we let

$$
\begin{aligned}
& (x(\theta), y(\theta)) \\
& = \begin{cases}\left(x_{c}+A \cos \theta-B \sin \theta, y_{c}+A \cos \theta+B \sin \theta\right) & \text { if } \vartheta=\frac{\pi}{4}, \\
\left(x_{c}+A \cos \theta+B \sin \theta, y_{c}-A \cos \theta+B \sin \theta\right) & \text { if } \vartheta=-\frac{\pi}{4},\end{cases}
\end{aligned}
$$

where $(A, B)=((\sqrt{2} / 2) a,(\sqrt{2} / 2) b)$. By the previous discussions, if $\alpha=\beta=\mu /(2+\rho)$, then $\Gamma_{\mu, \rho,-\mu^{2} /(2+\rho)}$ where
$f=\Gamma_{\mu, \rho}(\mu /(2+\rho), \mu /(2+\rho))$ is a point ellipse and in view of (49), we may arrive at the following result.

Theorem 15. Suppose $|\rho|<2$ and $(\alpha, \beta) \neq(\mu /(2+$ $\rho), \mu /(2+\rho))$ such that $f=\Gamma_{\mu, \rho}(\alpha, \beta)$. Let $\left\{\left(x\left(\theta_{k}\right), y\left(\theta_{k}\right)\right)\right\}_{k \in \mathbf{N}}$ be generated by the Tracking Procedure with input $(\alpha, \beta)=$ $\left(x\left(\theta_{0}\right), y\left(\theta_{0}\right)\right)$. For any $m \in \mathbf{N}$,

$$
\theta_{m+1}-\theta_{m}=\arccos \left(-\frac{\rho}{2}\right)
$$

Proof. In view of (49), we first consider the case where $\vartheta=$ $\pi / 4$. By (49), it follows that

$$
\begin{aligned}
x_{k} & =x_{c}+A \cos \theta_{k}-B \sin \theta_{k}, \\
x_{k+1} & =y_{c}+A \cos \theta_{k}+B \sin \theta_{k},
\end{aligned}
$$

as well as

$$
\begin{aligned}
& x_{k+1}=x_{c}+A \cos \theta_{k+1}-B \sin \theta_{k+1}, \\
& x_{k+2}=y_{c}+A \cos \theta_{k+1}+B \sin \theta_{k+1} .
\end{aligned}
$$

Note that $x_{c}=y_{c}=\mu /(2+\rho)$. By (7) and (52), we also have

$$
\begin{aligned}
x_{k+2}= & \mu-\rho x_{k+1}-x_{k} \\
= & \mu-\rho\left(y_{c}+A \cos \theta_{k+1}-B \sin \theta_{k+1}\right) \\
& -\left(x_{c}+A \cos \theta_{k}-B \sin \theta_{k}\right) \\
= & y_{c}+A\left(-\rho \cos \theta_{k+1}-\cos \theta_{k}\right) \\
& +B\left(\sin \theta_{k}+\rho \sin \theta_{k+1}\right) .
\end{aligned}
$$

In view of (52), with respect to $x_{k+2}$, it follows that

$$
\left(-\rho \cos \theta_{k+1}-\cos \theta_{k}\right)^{2}+\left(\sin \theta_{k}+\rho \sin \theta_{k+1}\right)^{2}=1,
$$

which leads to

$$
\begin{aligned}
& \cos \theta_{k+1} \cos \theta_{k}+\sin \theta_{k+1} \sin \theta_{k}=\cos \left(\theta_{k+1}-\theta_{k}\right) \\
& =-\frac{\rho}{2} .
\end{aligned}
$$

The case where $\vartheta=-\pi / 4$ can be handled similarly. Hence, (50) is true and this completes the proof.

By Theorem 15, we can find the necessary and sufficient condition for a solution $\varphi$ of (7) with $|\rho|<2$ to be a periodic sequence.

Corollary 16. Suppose $|\rho|<2$ and $(\alpha, \beta) \neq(\mu /(2+\rho), \mu /(2+$ $\rho)$ ) such that $f=\Gamma_{\mu, \rho}(\alpha, \beta)$. Let $\left\{\left(x_{k}, y_{k}\right)\right\}_{k \in \mathbf{N}}$ be generated by the Tracking Procedure with input $\left(x_{0}, y_{0}\right)=(\alpha, \beta)$. Then $\left\{\left(x_{k}, y_{k}\right)\right\}_{k \in \mathbf{N}}$ is periodic if and only if

$$
\frac{1}{\pi} \arccos \left(-\frac{\rho}{2}\right) \in \mathbf{Q}
$$

Furthermore, suppose $\pi^{-1} \arccos (-\rho / 2)=(m / n) \in \mathbf{Q}$ such that $m / n$ is irreducible and $0<m<n$. If $m$ is even, then $\left\{\left(x_{k}, y_{k}\right)\right\}_{k \in \mathbf{N}}$ is n-periodic; otherwise, $\left\{\left(x_{k}, y_{k}\right)\right\}_{k \in \mathbf{N}}$ is $2 n$ periodic. 
Proof. Note that if $\rho \neq-2$ and $(\alpha, \beta)=(\mu /(2+\rho), \mu /(2+\rho))$, then by the Tracking Procedure and Proposition $1,\left(x_{1}, y_{1}\right)=$ $(\alpha, \beta)$ which implies $\left(x_{k}, y_{k}\right)=(\alpha, \beta)$ for all $k \in \mathbf{N}$. Suppose $|\rho|<2$ and $(\alpha, \beta) \neq(\mu /(2+\rho), \mu /(2+\rho))$. By (49), we have

$$
\begin{aligned}
& \left(x_{k}, y_{k}\right)=\left(x_{c}+A \cos \theta_{k}-B \cos \theta_{k}, y_{c}+A \cos \theta_{k}\right. \\
& \left.\quad+B \sin \theta_{k}\right) .
\end{aligned}
$$

By Theorem 15 , for any $k \in \mathbf{N}, \theta_{\Delta}=\theta_{k+1}-\theta_{k}$ is constant such that $\cos \left(\theta_{\Delta}\right)=-\rho / 2$. Hence $\theta_{k}=\theta_{0}+k \theta_{\Delta}$. If $\left\{\left(x_{k}, y_{k}\right)\right\}_{k \in \mathbf{N}}$ is periodic with period $n \in \mathbf{Z}^{+}$, then we see that there is some $m \in \mathbf{Z}^{+}$such that $\theta_{n}=\theta_{0}+2 m \pi$. Accordingly, $n \theta_{\Delta}=2 m \pi$ which implies

$$
\frac{\theta_{\Delta}}{\pi}=\frac{1}{\pi} \arccos \left(-\frac{\rho}{2}\right)=\frac{m}{n} \in \mathbf{Q}
$$

On the other hand, if (56) holds, then, by the previous discussions and (58), $\left\{\left(x_{k}, y_{k}\right)\right\}_{k \in \mathbf{N}}$ is periodic with period $2 n$. Note that by Propositions 2 and 4 , if $\left\{\left(x_{k}, y_{k}\right)\right\}_{k \in \mathbf{N}}$ is $\omega$ periodic with $(\alpha, \beta) \neq(\mu /(2+\rho), \mu /(2+\rho))$, then $\omega>2$. Next, suppose $r \in \mathbf{Q}$, where $r=\pi^{-1} \arccos (-\rho / 2)$. Since $2 j \pi$ where $j \in \mathbf{Z}$ are periods of $\cos (x)$, we can first restrict ourselves to $0<r<2$, where $r \neq \pi$. Also, if $\pi<x<2 \pi$, then $\cos (x)=\cos (-x)=\cos (2 \pi-x)$ and this allows us to further concentrate on $0<r<1$. Note that $\cos (x)$ is bijective on $(0, \pi)$. Hence, for each $\rho$ such that $r \in \mathbf{Q}$, where $0<r<1$, there is a unique pair $(m, n)$ such that $r=(m / n)$, where $m$ and $n$ are coprime. In view of (58), it is easy to see that if $m$ is even, then $n$ is a period of $\left\{\left(x_{k}, y_{k}\right)\right\}_{k \in \mathbf{N}}$; if $m$ is odd, then $\left\{\left(x_{k}, y_{k}\right)\right\}_{k \in \mathbf{N}}$ is periodic with period $2 n$. In the former case, we claim that $\left\{\left(x_{k}, y_{k}\right)\right\}_{k \in \mathrm{N}}$ is $q$-periodic. To this end, if $q^{\prime}<q$ is a period of $\left\{\left(x_{k}, y_{k}\right)\right\}_{k \in \mathbf{N}}$, then, by the previous discussions, there is $p^{\prime} \in \mathbf{Z}^{+}$such that $p^{\prime}$ as well $q^{\prime}$ are coprime and $r=p^{\prime} / q^{\prime}$. Hence, $\left(p^{\prime} / q^{\prime}\right)=(p / q)$ which is not possible. The latter case can be handled similarly and this completes the proof.

In view of Corollary 16, we have found the necessary and sufficient condition for $\left\{\left(x_{k}, y_{k}\right)\right\}_{k \in \mathbf{N}}$, which is generated by the Tracking Procedure with input $\left(x_{0}, y_{0}\right) \neq(\mu /(2+\rho), \mu /(2+$ $\rho))$ such that $f=\Gamma_{\mu, \rho}\left(x_{0}, y_{0}\right)$ and $|\rho|<2$, to be periodic. Let $\rho=-1,0$, or 1 ; for instance, by $(58)$, we take $(m / n)=1 / 3$, $1 / 2$, or $2 / 3$, correspondingly, so that $\left\{\left(x_{k}, y_{k}\right)\right\}_{k \in \mathbf{N}}$ is 6-, 4-, or 3 -periodic, respectively. For another example, by (58) again, if $(m / n)=2 / 5$ or $4 / 5$, then $\left\{\left(x_{k}, y_{k}\right)\right\}_{k \in \mathbf{N}}$ is 5-periodic where $\rho=(1-\sqrt{5}) / 2$ or $\rho=(1+\sqrt{5}) / 2$, respectively.

For the analysis of nonnegative or negative solutions of (7) with $|\rho|<2$, we need one more result.

Theorem 17. Let $r \notin \mathbf{Q}$. Suppose $\phi_{k} \equiv 2 \pi k r \bmod 2 \pi$ where $k \in \mathbf{N}$. Then $\left\{\phi_{k} \mid k \in \mathbf{N}\right\}$ is dense in $[0,2 \pi]$.

In view of Theorem 17 , let $\eta_{k} \equiv k r \bmod 1$, where $k \in$ $\mathbf{N}$. Then we see that $\eta_{k}$ is dense in $[0,1]$ (see the details in [12] in pages 280 and 281). Suppose $s \in[0,2 \pi]$. Since $(s / 2 \pi) \in[0,1]$, there exists a subsequence $\left\{\eta_{k_{j}}\right\}_{j \in \mathbf{N}}$ of $\left\{\eta_{k}\right\}_{k \in \mathbf{N}}$ such that $\eta_{k_{j}}=s / 2 \pi$, when $j \rightarrow \infty$, from which it follows that

$$
\lim _{j \rightarrow \infty} 2 \pi \eta_{k_{j}}=s .
$$

Accordingly, $\phi_{k}$ is dense in $[0,2 \pi]$ as desired.

Recall that by the Principal Axes Theorem, $\bar{\Pi}$ and $\bar{\Pi}_{\perp}$ are the principal axes of $\Gamma_{\mu, \rho, f}$, where $\rho \neq-2$ and $f=\Gamma_{\mu, \rho}(\alpha, \beta)$, $(\alpha, \beta) \in \mathbf{R}^{2}$. For $-2<\rho<0$ and $(\alpha, \beta) \in \mathbf{R}^{2}$ such that $f=$ $\Gamma_{\mu, \rho}(\alpha, \beta)$, one property of $\left\{\left(x_{k}, y_{k}\right)\right\}_{k \in \mathbf{N}}$ which is generated by the Tracking Procedure with input $\left(x_{0}, y_{0}\right)=(\alpha, \beta) \neq(\mu /(2+$ $\rho), \mu /(2+\rho))$ is revealed as follows.

Corollary 18. Suppose $-2<\rho<0$ and $(\alpha, \beta) \neq(\mu /(2+$ $\rho), \mu /(2+\rho))$ such that $f=\Gamma_{\mu, \rho}(\alpha, \beta)$. Let $\left\{\left(x_{k}, y_{k}\right)\right\}_{k \in \mathbf{N}}$ be generated by the Tracking Procedure with input $\left(x_{0}, y_{0}\right)=$ $(\alpha, \beta)$. The set $\left\{\left(x_{m}, y_{m}\right) \mid m \in \mathbf{N}\right\}$ cannot lie entirely in one of the half planes divided by $\bar{\Pi}_{\perp}$.

Proof. Since $-2<\rho<0$ and $(\alpha, \beta) \neq(\mu /(2+\rho), \mu /(2+\rho))$, $\Gamma_{\mu, \rho, f}$ is an ellipse. By Corollary 16 and Propositions 2 and 4, if $\pi^{-1} \arccos (-\rho / 2) \in \mathbf{Q}$, then $\varphi$ is $\omega$-periodic, where $\omega \geq 3$; otherwise, $\varphi$ is aperiodic. Suppose $\pi^{-1} \arccos (-\rho / 2) \in \mathbf{Q}$. Then $\left\{\left(x_{k}, y_{k}\right)\right\}_{k \in \mathbf{N}}$ is $\omega$-periodic, where $\omega \geq 3$ such that $\left(x_{\omega}, y_{\omega}\right)=(\alpha, \beta)$. First of all, by the Tracking Procedure, we see that $\left(x_{k}, y_{k}\right)$ moves on $\Gamma_{\mu, \rho, f}$ in a clockwise manner. Secondly, $\Gamma_{\mu, \rho, f}$ is symmetric with respect to $x=y$. Hence, without loss of generality, we suppose $\Pi(\alpha, \beta) \geq 0$. Since $\left(x_{k}, y_{k}\right)$ move on $\Gamma_{\mu, \rho, f}$ in a clockwise manner; by the Tracking Procedure, we can be sure that for some $i \in\{0,1,2, \ldots, \omega-1\}$, $H\left(\Pi_{\perp}\left(x_{i}, y_{i}\right)\right) H\left(\Pi_{\perp}(\alpha, \beta)\right)<0$. Note that if $H\left(\Pi_{\perp}(\alpha, \beta)\right)=0$, then we may investigate the orbit $\mathcal{O}^{+}\left(E^{-1} \varphi\right)$ or $\mathcal{O}\left(E^{1} \varphi\right)$ so that $H\left(\Pi_{\perp}(\beta, \mu-\rho \beta-\alpha)\right) \neq 0$ or $H\left(\Pi_{\perp}(\mu-\rho \alpha-\beta, \alpha)\right) \neq 0$, respectively. Suppose $\pi^{-1} \arccos (-\rho / 2) \notin \mathbf{Q}$. Without loss of generality, we suppose $\Pi_{\perp}(\alpha, \beta)<0$ and $(s, t) \in \Gamma_{\mu, \rho, f}$ such that $\Pi_{\perp}(s, t)>0$. Then by Theorem 17, there is a subsequence $\left\{\left(x_{k_{i}}, y_{k_{i}}\right)\right\}_{i \in \mathbf{N}}$ such that $\lim _{i \rightarrow \infty}\left(x_{k_{i}}, y_{k_{i}}\right)=(s, t)$ as required and this completes the proof.

The previous discussions about the asymptotic behaviors of the solution of (7) help us to understand the periodicity of $\varphi$. To further analyze the positivity of solutions of (7), we require one specific solution ${ }_{\rho}^{\mu} \chi=\left\{{ }_{\rho}^{\mu} \chi_{k}\right\}_{k \in \mathbf{Z}}$ with $\mu \neq 0, \rho<2$, and $\left({ }_{\rho}^{\mu} \chi_{0},{ }_{\rho}^{\mu} \chi_{1}\right)=(\mu / 2,0)$. We note that $f=\Gamma_{\mu, \rho}(\mu / 2,0)=$ $-\mu^{2} / 4$. Also, some properties of ${ }_{\rho}^{\mu} \chi$ are useful for the ensuing analysis and they are as follows.

Proposition 19. For $\mu, \rho \in \mathbf{R}$, the lines $y=0$ and $x=0$ intercept with $\Gamma_{\mu, \rho,-\mu^{2} / 4}$ at $(\mu / 2,0)$ and $(0, \mu / 2)$, respectively. Furthermore, if $\rho<2$ and $\mu \neq 0$, then $\Gamma_{\mu, \rho,-\mu^{2} / 4}$ is a nondegenerate conic section and the lines $y=0$ and $x=0$ are tangents of $\Gamma_{\mu, \rho,-\mu^{2} / 4}$ at the points $(\mu / 2,0)$ and $(0, \mu / 2)$, respectively.

Proof. First of all, we show that $\Gamma_{\mu, \rho,-\mu^{2} / 4}$ meets the $y=0$ line at $(\mu / 2,0)$. To this end, note that

$$
\Gamma_{\mu, \rho,-\mu^{2} / 4}(x, 0)=x^{2}-\mu x+\frac{1}{4} \mu^{2}=\left(x-\frac{1}{2} \mu\right)^{2}=0
$$




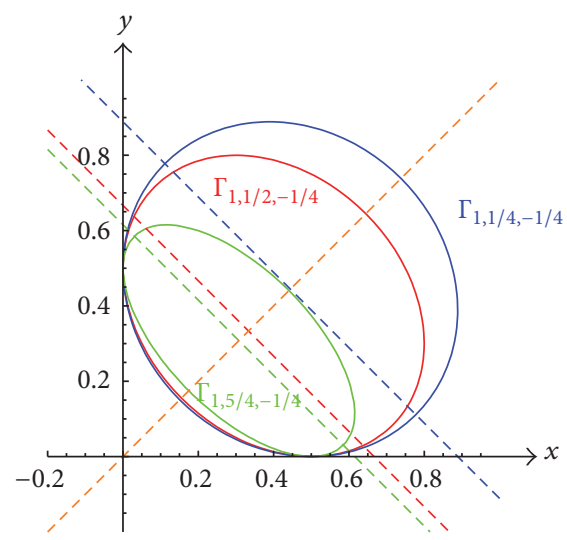

Figure 16: The $\Gamma_{1, \rho,-1 / 4}$, where $0<\rho<2$.

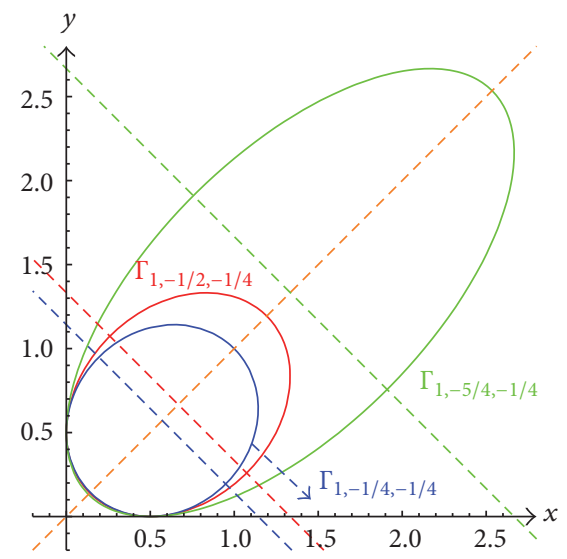

FIGURE 17: The $\Gamma_{1, \rho,-1 / 4}$, where $-2<\rho<0$.

and, thus, $x=\mu / 2$ is the unique solution to $\Gamma_{\mu, \rho,-\mu^{2} / 4}$ and $y=0$. Accordingly, the $y=0$ line intercept with $\Gamma_{\mu, \rho,-\mu^{2} / 4}$ at $(\mu / 2,0)$. By the previous discussions, if $\rho=2$, then $\Gamma_{\mu, 2,-\mu^{2} / 4}$ is a degenerate parabola made up of the $x+y-(1 / 2) \mu=0$ line; if $\rho=-2$ and $\mu=0$, then $\Gamma_{0,-2,0}$ is a degenerate parabola made up of the $x+y=0$ line. Note that the $\Gamma_{\mu,-2,-\mu^{2} / 4}$ is a parabola with $\mu \neq 0$. Accordingly, if $\rho<2$ and $\mu \neq 0$, then $\Gamma_{\mu, \rho,-\mu^{2} / 4}$ is not a degenerate conic section which implies $\Gamma_{\mu,-2,-\mu^{2} / 4}$ is tangent to $x=0$ and $y=0$ at $(0, \mu / 2)$ as well as $(\mu / 2,0)$, respectively. The proof is complete.

We illustrate Proposition 19 by several plots of $\Gamma_{1, \rho,-\mu^{2} / 4}$, namely, ellipses with $0<\rho<2$ plotted in Figure 16, ellipses with $-2<\rho<0$ plotted in Figure 17, and hyperbola with $\rho<-2$ plotted in Figure 18.

In the following section, we establish the relation between the nonnegative (or negative) solution of (7) and a special solution ${ }_{\rho}^{\mu} \chi=\left\{{ }_{\rho}^{\mu} \chi_{k}\right\}_{k \in \mathbf{N}}$ in the geometric setting.

\section{A Special Positive Orbit}

Let ${ }_{\rho}^{\mu} \chi=\left\{{ }_{\rho}^{\mu} \chi\right\}_{k \in \mathbf{Z}}$ be the solution of (7) with $\left({ }_{\rho}^{\mu} \chi_{0},{ }_{\rho}^{\mu} \chi_{1}\right)=(\mu /$ $2,0)$. By (12) and (13), we see that $U\left({ }_{\rho}^{\mu} \chi\right)=E^{-2}\left({ }_{\rho}^{\mu} \chi\right)$ which

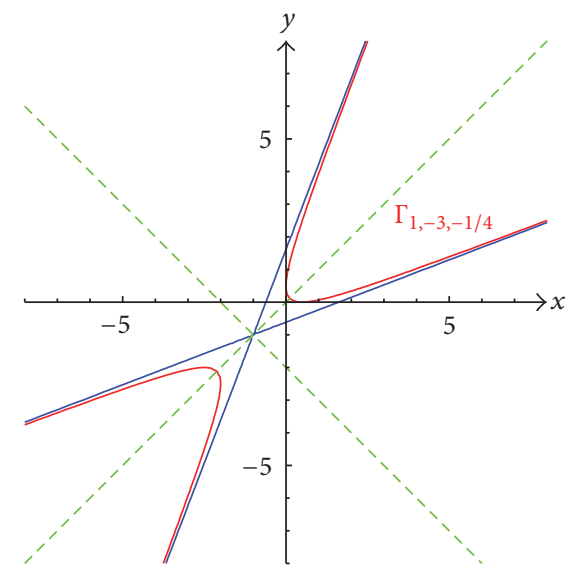

FIGURE 18: The plot of $\Gamma_{1, \rho,-1 / 4}$, where $\rho=-3$.

implies ${ }_{\rho}^{\mu} \chi_{k}={ }_{\rho}^{\mu} \chi_{-k+2}$ for all $m \in \mathbf{Z} \backslash \mathbf{N}$. Hence, it is found that $\mathcal{O}_{\perp}^{+}\left({ }_{\rho}^{\mu} \chi\right) \subset \mathcal{O}^{+}\left({ }_{\rho}^{\mu} \chi\right)$ and by $(15)$, we have

$$
\begin{aligned}
& \mathcal{O}\left({ }_{\rho}^{\mu} \chi\right)=\mathcal{O}^{+}\left({ }_{\rho}^{\mu} \chi\right) \cup\left\{\left(x_{k}, y_{k}\right)=\left(v_{k}, u_{k}\right) \mid\left(u_{k}, v_{k}\right)\right. \\
& \left.\in \mathcal{O}^{+}\left({ }_{\rho}^{\mu} \chi\right), k \in \mathbf{N}\right\} .
\end{aligned}
$$

Note that (61) allows us to obtain $\mathcal{O}\left({ }_{\rho}^{\mu} \chi\right)$ from plotting $\mathcal{O}^{+}\left({ }_{\rho}^{\mu} \chi\right)$ only. In the sequel, we focus on the the "positive" orbit of $\mathcal{O}^{+}\left({ }_{\rho}^{\mu} \chi\right)$ when the orbit $\mathcal{O}\left({ }_{\rho}^{\mu} \chi\right)$ is discussed. By the Tracking Procedure with $\left({ }_{\rho}^{\mu} \chi_{0},{ }_{\rho}^{\mu} \chi_{1}\right)=(\mu / 2,0)$, we can generate the positive orbit $\mathcal{O}^{+}\left({ }_{\rho}^{\mu} \chi\right)$. Next, we establish the connection between the nonnegative solutions and the specific solution ${ }_{\rho}^{\mu} \chi$.

Let

$\Phi_{\mu, \rho}$

$=\bigcup\{\mathcal{O}(\xi) \mid \xi$ is a nonnegative solution of $(7)\}$,

$\Omega_{\mu, \rho}=\bigcup\{\mathcal{O}(\xi) \mid \xi$ is a negative solution of $(7)\}$.

Let $\varphi=\left\{\varphi_{k}\right\}_{k \in \mathbf{Z}}$ of (7) with $\left(\varphi_{m}, \varphi_{m+1}\right)=(\alpha, \beta)$ for some $m \in$ $Z$. Then by the definitions of $\Phi_{\mu, \rho}$ and $\Omega_{\mu, \rho}$, we can be sure that

(1) $\varphi$ is a nonnegative solution if and only if $(\alpha, \beta) \in \Phi_{\mu, \rho}$;

(2) $\varphi$ is a negative solution if and only if $(\alpha, \beta) \in \Omega_{\mu, \rho}$.

Now, it is our objective to find $\Phi_{\mu, \rho}$ and $\Omega_{\mu, \rho}$ and in several special cases where $\rho<2$ and $\mu>0$, we will show that $\Phi_{\mu, \rho}$ and $\Omega_{\mu, \rho}$ can be expressed as the intersections of certain half planes in $\mathbf{R}^{2}$.

Proposition 20. Let $\varphi=\left\{\varphi_{k}\right\}_{k \in \mathbf{Z}}$ and $c=\left\{c_{k}\right\}_{k \in \mathbf{Z}}$ be solutions of (7) with $\left(\varphi_{0}, \varphi_{1}\right)=(\alpha, \beta)$ and $\left(c_{0}, c_{1}\right)=(0,0)$. Let $a=$ $\left\{a_{k}\right\}_{k \in \mathbf{Z}}$ and $b=\left\{b_{k}\right\}_{k \in \mathbf{Z}}$ be solutions of (7) with $\mu=0$, with $\left(a_{0}, a_{1}\right)=(0,1)$, and with $\left(b_{0}, b_{1}\right)=(1,0)$. Then we have

$$
\varphi_{k}=b_{k} \alpha+a_{k} \beta+c_{k} .
$$


Proof. For the sake of convenience, we suppose $k \in \mathbf{N}$. First of all, if $\left(a_{0}, b_{0}, c_{0}\right)=(0,1,0)$ and $\left(a_{1}, b_{1}, c_{1}\right)=(1,0,0)$, then $\left(\varphi_{0}, \varphi_{1}\right)=(\alpha, \beta)$ which implies (63) is true when $k \leq 1$. Next, we show that (63) holds for $k \geq 2$. To this end, by (7), it follows that

$$
\begin{aligned}
\varphi_{k+2}= & \mu-\rho \varphi_{k+1}-\varphi_{k}, \\
= & \mu-\rho\left(b_{k+1} \alpha+a_{k+1} \beta+c_{k+1}\right) \\
& -\left(b_{k} \alpha+a_{k} \beta+c_{k}\right) \\
= & \left(-\rho b_{k+1}-b_{k}\right) \alpha+\left(-\rho a_{k+1}-a_{k}\right) \beta \\
& +\left(\mu-\rho c_{k+1}-c_{k}\right) \\
= & b_{k+2} \alpha+a_{k+2} \beta+c_{k+2},
\end{aligned}
$$

as required. Note that in view of (66), as well as (67), we have

$$
\begin{aligned}
& a_{k+2}=-\rho a_{k+1}-a_{k}, \\
& b_{k+2}=-\rho b_{k+1}-b_{k}, \\
& c_{k+2}=\mu-\rho c_{k+1}-c_{k} .
\end{aligned}
$$

Next, we consider the case where $k \in \mathbf{Z} \backslash \mathbf{N}$. Let $\psi=U E^{-1} \varphi$ such that $\left(\psi_{0}, \psi_{1}\right)=(\beta, \alpha)$. Thus, we may reformulate (63) for $\psi_{m}$, where $m \in \mathbf{N}$ as

$$
\psi_{m}=a_{k} \alpha+b_{k} \beta+c_{k} .
$$

Furthermore, in view of (68), $U E^{-1} a=b$ and $U E^{-1} c=c$ which implies (63) is true and this completes the proof.

With $\left(a_{k}, b_{k}, c_{k}\right)$ defined in Proposition 20, we define the 2 -variable function

$$
h_{\mu, \rho, k}(x, y)=a_{k} x+b_{k} y+c_{k}
$$

and the set

$$
\bar{h}_{\mu, \rho, k}(x, y)=\left\{(x, y) \in \mathbf{R}^{2} \mid a_{k} x+b_{k} y+c_{k}=0\right\} .
$$

Then we construct a set which is an intersection of half planes:

$$
\mathscr{H}_{\mu, \rho}=\bigcap_{k \in \mathbf{Z}}\left\{(x, y) \in \mathbf{R}^{2} \mid h_{\mu, \rho, k}(x, y) \geq 0\right\} .
$$

We claim that

$$
\Phi_{\mu, \rho}=\mathscr{H}_{\mu, \rho} .
$$

Let $\varphi=\left\{\varphi_{k}\right\}_{k \in \mathbf{Z}}$ be a solution of (7). Note that by Corollaries 3 and 5 and the definition of (72), we see that $\Phi_{\mu, \rho}$ is nonempty which implies $\mathscr{H}_{\mu, \rho} \neq \emptyset$. If $\left(\varphi_{m}, \varphi_{m+1}\right) \in \Phi_{\mu, \rho}$, for some $m \in$ $\mathbf{Z}$, then, by the definition, $\varphi$ is nonnegative which implies $\varphi_{n} \geq$ 0 for all $n \in \mathbf{Z}$. Since each $h_{\mu, \rho, k}$ is characterized by $\varphi_{k}$, in view of (72), we see that $\left(\varphi_{n}, \varphi_{n+1}\right) \in \mathscr{H}_{\mu, \rho}$. On the other hand, if $\left(\varphi_{i}, \varphi_{i+1}\right) \notin \Phi_{\mu, \rho}$, then $\varphi$ is not nonnegative which implies there is some $j \in \mathbf{Z}$ such that $\varphi_{j}<0$. Accordingly, we have $h_{\mu, \rho, j}(x, y)<0$ and by (72), it follows that $\mathscr{H}_{\mu, \rho}=\emptyset$ which is not possible.
Theoretically, we can calculate $h_{\mu, \rho, k}(x, y)$ for all $k \in \mathbf{Z}$ to determine (73) explicitly. Suppose $\varphi=\left\{\varphi_{k}\right\}_{k \in \mathbf{Z}}$ is an $\omega$ periodic solution of (7) with given $\mu$ and $\rho$. Then we only have to calculate $h_{\mu, \rho, k}(x, y)$, where $k \in\{0,1,2, \ldots, \omega-1\}$, to determine $\Phi_{\mu, \rho}\left(\right.$ or $\left.\Omega_{\mu, \rho}\right)$. Even though this is feasible for us, it is still difficult to illustrate $\Phi_{\mu, \rho}$ explicitly on $\mathbf{R}^{2}$ when $\omega$ is sufficiently large. In the sequel, we find $\Phi_{\mu, \rho}$, where $\rho<2$ and $\mu \neq 0$, by one specific $\mathcal{O}\left({ }_{\rho}^{\mu} \chi\right) \subseteq \Gamma_{\mu, \rho,-\mu^{2} / 4}$. Note that the cases where $\Phi_{\mu, \rho}$, with $\rho \geq 2$ or $\mu=0$, can be handled separately. By means of investigating the specific $\mathcal{O}\left({ }_{\rho}^{\mu} \chi\right)$ and $\Gamma_{\mu, \rho,-\mu^{2} / 4}$ where $\rho<2$ and $\mu \neq 0, \Phi_{\mu, \rho}$ can be found in an explicit way. Recall that if $\rho<2$ and $\mu \neq 0$, then the $x$ axis and the $y$-axis are tangent to $\Gamma_{\mu, \rho,-\mu^{2} / 4}$ at $(0, \mu / 2)$ as well as $(\mu / 2,0)$, respectively. Let ${ }_{\rho}^{\mu} \chi=\left\{{ }_{\rho}^{\mu} \chi_{k}\right\}_{k \in \mathbf{Z}}$ be a solution of (7) with $\left({ }_{\rho}^{\mu} \chi_{0},{ }_{\rho}^{\mu} \chi_{1}\right)=(\mu / 2,0)$. The tangent line $\bar{l}_{\mu, \rho, k}(x, y)$ of $\Gamma_{\mu, \rho,-\mu^{2} / 4}$ at $\left(x_{k}, y_{k}\right)$ is given by $l_{\mu, \rho, k}(x, y)=0$, where

$$
\begin{aligned}
l_{\mu, \rho, k}(x, y)= & \left(2 x_{k}+\rho y_{k}-\mu\right) x+\left(\rho x_{k}+2 y_{k}-\mu\right) y \\
& +\left(\frac{1}{2} \mu^{2}-\mu x_{k}-\mu y_{k}\right) .
\end{aligned}
$$

In addition, we also let $\bar{l}_{\mu, \rho, k}^{\prime}$ be the line so that $\bar{l}_{\mu, \rho, k}^{\prime}$ and $\bar{l}_{\mu, \rho, k}$ are symmetric with respect to the $x=y$ line. Now, we establish the connection between $\mathscr{H}_{\mu, \rho}$ and the set of intersection of half planes divided by $\bar{l}_{\mu, \rho, k}(x, y)$.

Theorem 21. Given $\rho, \mu \in \mathbf{R}$, where $\rho<2$ and $\mu>0$, then we have

$$
\begin{array}{r}
\mathscr{H}_{\mu, \rho}=\bigcap_{k \in \mathbf{N}}\left\{(x, y) \in \mathbf{R}^{2} \mid l_{\mu, \rho, k}(x, y) \leq 0\right\} \\
\cup\left\{(x, y) \in \mathbf{R}^{2} \mid l_{\mu, \rho, k}^{\prime}(x, y) \leq 0\right\} .
\end{array}
$$

Proof. First of all, we consider the case where $k \geq 0$ and we show that $h_{\mu, \rho, k}(x, y)=r l_{\mu, \rho, k}(x, y)$, where $r=\mu(\rho-2) / 2<0$. To this end, we show the following results,

$$
\begin{aligned}
& r a_{k}=\left(2 x_{k}+\rho y_{k}-\mu\right), \\
& r b_{k}=\left(\rho x_{k}+2 y_{k}-\mu\right), \\
& r c_{k}=\left(\frac{1}{2} \mu^{2}-\mu x_{k}-\mu y_{k}\right),
\end{aligned}
$$

are true for all $k \in \mathbf{Z}$. Let $\left\{\left(x_{k}, y_{k}\right)\right\}_{k \in \mathbf{N}}$ be $\mathcal{O}^{+}\left({ }_{\rho}^{\mu} \chi\right)$ with $\left(x_{0}, y_{0}\right)=\left({ }_{\rho}^{\mu} \chi_{0},{ }_{\rho}^{\mu} \chi_{1}\right)=(\mu / 2,0)$. Since $\left(a_{0}, b_{0}, c_{0}\right)=(0,1,0)$ and $\left(a_{1}, b_{1}, c_{1}\right)=(1,0,0)$, by (70) and (74), it follows that (75) holds for $m \leq 1$. Now, we suppose that for some $j \in \mathbf{N}$ such that (75) holds for $j$ and $j+1$. Then we have seen that for $i \in\{j, j+1\}$

$$
\begin{aligned}
& r\left(a_{i}, b_{i}, c_{i}\right) \\
& \quad=\left(2 x_{i}+\rho y_{i}-\mu, \rho x_{i}+2 y_{i}-\mu, \frac{1}{2} \mu^{2}-x_{i}-y_{i}\right) .
\end{aligned}
$$


For $c_{j+2}$, by (68), (76), and (7), it follows that

$$
\begin{aligned}
r c_{j+2}= & r\left(\mu-\rho c_{j+1}-c_{j}\right) \\
= & r \mu-\rho r c_{j+1}-r c_{j} \\
= & r \mu-\rho\left(\frac{1}{2} \mu^{2}-\mu x_{j+1}-\mu y_{j+1}\right) \\
& -\left(\frac{1}{2} \mu^{2}-\mu x_{j}-\mu y_{j}\right) \\
= & \frac{1}{2} \rho \mu^{2}-\mu^{2}-\frac{1}{2} \rho \mu^{2}-\frac{1}{2} \mu^{2}+\mu\left(\rho x_{j+1}+x_{j}\right) \\
& +\mu\left(\rho y_{j+1}+y_{j}\right) \\
= & \frac{1}{2} \mu^{2}-\mu\left(\mu-\rho x_{j+1}-x_{j}\right) \\
& -\mu\left(\mu-\rho y_{j+1}-y_{j}\right) \\
= & \frac{1}{2} \mu^{2}-\mu x_{j+2}-\mu y_{j+2},
\end{aligned}
$$

as required. Next we consider $a_{j+2}$ and $b_{j+2}$. By (68), (77), and (7) again, in the former case,

$$
\begin{aligned}
r a_{j+2} & =r\left(-\rho a_{j+1}-a_{j}\right) \\
& =-\rho r a_{j+1}-r a_{j} \\
& =-\rho\left(2 x_{j+1}+\rho y_{j+1}-\mu\right)-\left(2 x_{j}+\rho y_{j}-\mu\right) \\
& =-2\left(\rho x_{j+1}+x_{j}-\mu\right)-\rho\left(\rho y_{j+1}+y_{j}-\mu\right)-\mu \\
& =2 x_{j+2}+\rho y_{j+2}-\mu,
\end{aligned}
$$

as desired; the latter case can be handled similarly. By induction, we see that for all $m \in \mathbf{N}, h_{\mu, \rho, k}(x, y)=r l_{\mu, \rho, k}(x, y)$ is true. Now, we consider the case where $k<0$. By the reflection and translation invariances, we transform $\psi=$ $U E^{-1} \varphi$ in Proposition 20 and $\zeta=U E^{-1}\left({ }_{\rho}^{\mu} \chi\right)$ and by similarly applied arguments for $k \geq 0$, we see that $h_{\mu, \rho, k}(x, y)=$ $r l_{\mu, \rho, k}(x, y)$, for all $k<0$. Note that $U\left({ }_{\rho}^{\mu} \chi\right)=E^{-2}\left({ }_{\rho}^{\mu} \chi\right)$ which implies $l_{\mu, \rho, k}(x, y)=l_{\mu, \rho,-k+2}^{\prime}(x, y)$, where $k<0$. Accordingly, if $k \geq 0$, then $h_{\mu, \rho, k}(x, y)=r l_{\mu, \rho, k}(x, y)$; if $k<0$, then $h_{\mu, \rho, k}(x, y)=r l_{\mu, \rho, k}(x, y)=r l_{\mu, \rho,-k+2}^{\prime}(x, y)$. Finally, since $r<0$, we see that (75) holds and this completes the proof.

Corollary 22. Let $\varphi=\left\{\varphi_{k}\right\}_{k \in \mathbf{Z}}$ be a solution of (7) with $\left(\varphi_{m}\right.$, $\left.\varphi_{m+1}\right)=(\sigma, \tau)$, for some $m \in \mathbf{Z}$. If $(\sigma, \tau) \in \overline{\mathscr{H}}_{\mu, \rho}$, then there is some $n \in \mathrm{Z}$ such that $\varphi_{n}=0$.

Proof. By the definitions of $\mathscr{H}_{\mu, \rho}$ in (75), if $(\sigma, \tau) \in \overline{\mathscr{H}}_{\mu, \rho}$, then $(\sigma, \tau)$ is on the tangent line $\bar{l}_{j}(x, y)$ at $\left({ }_{\rho}^{\mu} \chi_{j},{ }_{\rho}^{\mu} \chi_{j+1}\right)$, for some $j \in \mathbf{Z}$. By (75) again, without loss of generality, we may further suppose that $(\sigma, \tau) \in \bar{l}_{i}(x, y)$, for some $i \in \mathbf{N}$. For the sake of convenience, we let $\delta=\mu-\rho \tau-\sigma$ and $\left({ }_{\rho}^{\mu} \chi_{i},{ }_{\rho}^{\mu} \chi_{i+1}\right)=(s, t)$ such that ${ }_{\rho}^{\mu} \chi_{i+2}=\mu-\rho t-s=u$. Since $(\sigma, \tau) \in \bar{l}_{i}(x, y)$, by (74), we have

$$
\begin{aligned}
l_{i}(\sigma, \tau)= & (\rho s+2 t-\mu) \tau+(2 s+\rho t-\mu) \sigma-\mu s-\mu t \\
& +\frac{1}{2} \mu^{2}=0 .
\end{aligned}
$$

Next, we show that $(\tau, \delta) \in \bar{l}_{i+1}(x, y)$. To this end, we first have

$$
\begin{aligned}
l_{i+1}(x, y)= & (\rho t+2 u-\mu) y+(2 t+\rho u-\mu) x-\mu t \\
& -\mu u+\frac{1}{2} \mu^{2} .
\end{aligned}
$$

We apply $(x, y)=(\tau, \delta)=(\tau, \mu-\rho \tau-\sigma)$ and $u=\mu-\rho t-s$ to (81) and by simplifying, it is found that $l_{i+1}(\tau, \delta)=l_{i}(\sigma, \tau)=0$ which implies $(\tau, \delta) \in \bar{l}_{i+1}(x, y)$ as desired. Since $\bar{l}_{0}$ and $\bar{l}_{1}$ are the $y=0$ and $x=0$ lines, respectively, by the previous discussions, there is some $n \in \mathbf{Z}$ such that $\varphi_{n}=0$ and this completes the proof.

In this section, we introduced the definitions of $\Phi_{\mu, \rho}$ (or $\Omega_{\mu, \rho}$ ) so that the necessary and sufficient conditions for a solution $\varphi$ of (7) to be nonnegative (or negative) can be obtained, respectively. Also, in Theorem 21, we discussed the specific solution ${ }_{\rho}^{\mu} \chi=\left\{{ }_{\rho}^{\mu} \chi_{k}\right\}_{k \in \mathbf{Z}}$ of (7) with $\left({ }_{\rho}^{\mu} \chi_{0},{ }_{\rho}^{\mu} \chi_{1}\right)=$ $(\mu / 2,0)$ and the intersection of the closed half planes generated by the tangent lines at $\left({ }_{\rho}^{\mu} \chi_{k},{ }_{\rho}^{\mu} \chi_{k+1}\right)$, for all $k \in \mathbf{Z}$. For the case where $\rho<2$ and $\mu>0$, we will show that some $\Phi_{\mu, \rho}$ can be expressed explicitly in terms of $\mathscr{H}_{\mu, \rho}$ in the sequel. Note that by Proposition 7 and Theorem 8 , some $\Phi_{\mu, \rho}$ and $\Omega_{\mu, \rho}$ with $\rho<2$ and $\mu<0$ can be derived from the results of the cases where $\rho<2$ and $\mu>0$. Hence, with all the results derived in the previous discussions together, we are ready to discuss the necessary and sufficient conditions for $\varphi$ to be nonnegative (or negative).

\section{Nonnegative and Negative Solutions when $|\rho|>2$}

Let $\varphi=\left\{\varphi_{k}\right\}_{k \in \mathbf{Z}}$ be a solution of (7) defined by $\left(\varphi_{0}, \varphi_{1}\right)=$ $(\alpha, \beta)$. In the last section, we have completed the discussions about the periodicity of $\varphi$ and the asymptotic behaviors of $\mathcal{O}^{+}(\varphi)$ which is generated by the Tracking Procedure with input $(\alpha, \beta)$. Note that $\left(\varphi_{k}, \varphi_{k+1}\right)=\left(x_{k}, y_{k}\right)=\left(x_{k}, x_{k+1}\right)$, where $\left\{\left(x_{k}, y_{k}\right)\right\}_{k \in \mathbf{N}}$ is generated by the Tracking Procedure with input $\left(x_{0}, y_{0}\right)=(\alpha, \beta)$. To facilitate the ensuing discussions, we define two sets $\Theta_{\mu, \rho}$ and $\Upsilon_{\mu, \rho}$ on $\mathbf{R}^{2}$ where

$$
\begin{aligned}
& \Theta_{\mu, \rho}=\left\{(x, y) \in \mathbf{R}^{2} \mid L_{0, \rho, f}^{+}(x, y) \geq 0, L_{0, \rho, f}^{-}(x, y)\right. \\
& \quad \leq 0, x \geq 0, y \geq 0\}, \\
& \Upsilon_{\mu, \rho}=\left\{(x, y) \in \mathbf{R}^{2} \mid L_{0, \rho, f}^{+}(x, y) \leq 0, L_{0, \rho, f}^{-}(x, y)\right. \\
& \quad \geq 0, x<0, y<0\} .
\end{aligned}
$$

Note that $\Theta_{\mu, \rho}$ is defined by four closed half planes and $\Upsilon_{\mu, \rho}$ is defined by two open as well as two closed half planes. 
Also note that by the definitions in (24) and (25), $L_{0, \rho, f}^{+}(x, y)$ and $L_{0, \rho, f}^{-}(x, y)$ are both independent of $f$. Now, we are able to apply these results to derive necessary and sufficient conditions for $\varphi$ to be nonnegative (or negative).

Theorem 23. Let $\varphi=\left\{\varphi_{k}\right\}_{k \in \mathbf{Z}}$ be a solution of (7) with $\rho>2$. Suppose $\left(\varphi_{m}, \varphi_{m+1}\right) \neq(\mu /(2+\rho), \mu /(2+\rho))$, for some $m \in$ $\mathrm{Z}$. Then $\varphi$ is neither a nonnegative solution nor a negative solution.

Proof. Without loss of generality, we let $m=0$. Let $\left(\varphi_{0}, \varphi_{1}\right)=$ $(\alpha, \beta)$ such that $f=\Gamma_{\mu, \rho}(\alpha, \beta)$. Let $\mathcal{O}^{+}(\varphi)=\left\{\left(x_{k}, y_{k}\right)\right\}_{k \in \mathbf{N}}$ and $\mathcal{O}_{\perp}^{+}(\varphi)=\left\{\left(s_{k}, t_{k}\right)\right\}_{k \in \mathbf{N}}$ be generated by the Tracking Procedure with inputs $\left(x_{0}, y_{0}\right)=(\alpha, \beta)$ and $\left(s_{0}, t_{0}\right)=(\beta, \alpha)$, respectively. Then $\Gamma_{\mu, \rho, f}$ is a hyperbola (or a degenerate one made up of two lines $\bar{L}_{\mu, \rho, f}^{+}$and $\left.\bar{L}_{\mu, \rho, f}^{-}\right)$such that $\mathcal{O}^{+}(\varphi), \mathcal{O}_{\perp}^{+}(\varphi) \subset \Gamma_{\mu, \rho, f}$. For the sake of convenience, we let $P_{c}=(\mu /(2+\rho), \mu /(2+\rho))$. If $(\alpha, \beta)=P_{c}$, then, by Proposition $2,\left(x_{k}, y_{k}\right)=\left(s_{k}, t_{k}\right)=P_{c}$, for all $k \in \mathbf{N}$. Suppose $(\alpha, \beta) \neq P_{c}$. By the previous discussions, we first consider the case where $(\alpha, \beta) \in \bar{L}_{\mu, \rho, f}^{+} \cup \bar{L}_{\mu, \rho, f}^{-} \backslash\left\{P_{c}\right\}$. If $(\alpha, \beta) \in \bar{L}_{\mu, \rho, f}^{+} \backslash\left\{P_{c}\right\}$, then $d\left((\alpha, \beta), \bar{L}_{\mu, \rho, f}^{+}\right) \leq d\left((\alpha, \beta), \bar{L}_{\mu, \rho, f}^{-}\right)$ and by Corollary 11, it is found that $x_{k} x_{k+1}=\varphi_{k} \varphi_{k+1}<0$ when $k \rightarrow \infty$; if $(\alpha, \beta) \in \bar{L}_{\mu, \rho, f}^{-}$, then we take $(\beta, \alpha) \in \bar{L}_{\mu, \rho, f}^{+}$ and we see that $(\beta, \alpha) \in O_{\perp}^{+}(\varphi)$ so that $s_{k} s_{k+1}<0$ when $k \rightarrow \infty$ as required. Next, suppose $\Gamma_{\mu, \rho, f}$ is a hyperbola. Then by Corollary 11 again, $x_{k} x_{k+1}=\varphi_{k} \varphi_{k+1}<0$ when $k \rightarrow \infty$. Accordingly, if $(\alpha, \beta) \neq P_{c}$, then $\varphi$ is neither a nonnegative nor a negative solution and this completes the proof.

Next, we consider the case where $\rho<-2$ and $\mu \in \mathbf{R}$. This case is a little more complicated than the one described in Theorem 23, and we give the result of $\mu=0$ first.

Theorem 24. Let $\varphi=\left\{\varphi_{k}\right\}_{k \in \mathbf{Z}}$ be a solution of (7) with $\rho<-2$ and $\mu=0$. Suppose $\left(\varphi_{m}, \varphi_{m+1}\right)=(\alpha, \beta)$, for some $m \in \mathbf{Z}$ such that $f=\Gamma_{\mu, \rho}(\alpha, \beta)$. Then $\varphi$ is a nonnegative solution if and only if $(\alpha, \beta) \in \Theta_{0, \rho}$; and $\varphi$ is a negative solution if and only if $(\alpha, \beta) \in \Upsilon_{0, \rho}$.

Proof. Note that in view of (82), $\Theta_{0, \rho}$ is symmetric with respect to the $x=y$ line and $\Theta_{0, \rho} \backslash\{(0,0)\}$ and $\Upsilon_{0, \rho}$ are symmetric with respect to the $x+y=0$ line. Let $\left\{\left(x_{k}\right.\right.$, $\left.\left.y_{k}\right)\right\}_{k \in \mathbf{N}}$ and $\left\{\left(s_{k}, t_{k}\right)\right\}_{k \in \mathbf{N}}$ be generated by the Tracking Procedure with inputs $\left(x_{0}, y_{0}\right)=(\alpha, \beta)$ and $\left(s_{0}, t_{0}\right)=(\beta, \alpha)$, respectively. Here, $\Gamma_{0, \rho, f}$, where $f=\Gamma_{0, \rho}(\alpha, \beta)$, is a hyperbola (or a degenerate one made up of two lines $L_{0, \rho, f}^{+}$and $L_{0, \rho, f}^{-}$). Note that $\Theta_{0, \rho}$ is a cone with two rays $L_{0, \rho, f}^{+}$and $L_{0, \rho, f}^{-}$ such that $u, v \geq 0$, where $(u, v) \in \Theta_{0, \rho}$. First of all, we show that $\Phi_{0, \rho}=\Theta_{0, \rho}$. To this end, we investigate $\left(x_{k}\right.$, $\left.y_{k}\right)$ and $\left(s_{k}, y_{k}\right)$ directly, and by Theorem 10, it is found that $\left(x_{k}, y_{k}\right),\left(s_{k}, t_{k}\right) \geq 0$ for all $k \in \mathbf{N}$ if and only if $\left(x_{0}, y_{0}\right) \in \Theta_{0, \rho}$. Accordingly, we have $\Phi_{0, \rho}=\Theta_{0, \rho}$ and the argument about $\Upsilon_{0, \rho}$ can be handled similarly. Note that both $\Theta_{0, \rho}$ and $\Theta_{0, \rho}$ are symmetric with respect to the $x=y$ line. Hence, the cases where $k \in \mathbf{Z} \backslash \mathbf{N}$ can be handled by the invariances of translation and reflection. The proof is complete.

Next, we discuss the case where $\rho<-2$ and $\mu \neq 0$.

Theorem 25. Let $\varphi=\left\{\varphi_{k}\right\}_{k \in \mathbf{Z}}$ be a solution of (7) with $\rho<-2$ and $\mu \neq 0$. Suppose $\left(\varphi_{m}, \varphi_{m+1}\right)=(\alpha, \beta)$ for some $m \in \mathbf{Z}$ such that $f=\Gamma_{\mu, \rho}(\alpha, \beta)$.

(i) Suppose $\mu>0$. Then $\varphi$ is a nonnegative solution if and only if $(\alpha, \beta) \in \mathscr{H}_{\mu, \rho}$ which is the intersection of the closed half planes defined in (75). Furthermore, $\varphi$ is a negative solution if and only if $(\alpha, \beta) \in \Upsilon_{\mu, \rho}$.

(ii) Suppose $\mu<0$. $\varphi$ is a nonnegative solution if and only if $(\alpha, \beta) \in \Theta_{\mu, \rho}$; and $\varphi$ is a negative solution if and only if $(\alpha, \beta) \in\left\{\mathscr{H}_{-\mu, \rho}^{\prime \prime} \backslash \partial \mathscr{H}_{-\mu, \rho}^{\prime \prime}\right\}$, where $\mathscr{H}_{-\mu, \rho}$ is the intersection of the closed half planes defined in (75).

Proof. Without loss of generality, we let $m=0$ and $P_{c}=$ $(\mu /(2+\rho), \mu /(2+\rho))$. Since $f=\Gamma_{\mu, \rho}(\alpha, \beta), \Gamma_{\mu, \rho, f}$ is a hyperbola (or a degenerate one, two lines $\bar{L}_{\mu, \rho, f}^{+}$and $\bar{L}_{\mu, \rho, f}^{+}$). Let $\mathcal{O}^{+}(\varphi)=$ $\left\{\left(x_{k}, y_{k}\right)\right\}_{k \in \mathbf{N}}$ and $\mathcal{O}_{\perp}^{+}=\left\{\left(s_{k}, t_{k}\right)\right\}_{k \in \mathbf{N}}$ be generated by the Tracking Procedure with inputs $\left(x_{0}, y_{0}\right)=(\alpha, \beta)$ and $\left(s_{0}, t_{0}\right)=$ $(\beta, \alpha)$, respectively. Suppose $\mu>0$. Then $\mu /(2+\rho)<0$. By Theorem 21, it follows that $\Phi_{\mu, \rho}=\mathscr{H}_{\mu, \rho}$ as desired. Next, by Corollary 11, if $(\alpha, \beta) \in \Upsilon_{\mu, \rho}$, then $x_{k}, s_{k}<0$, for all $k \in \mathbf{N}$. Conversely, if $x_{k}, s_{k}<0$ for all $k \in \mathbf{N}$, then, by Theorem 10 and Corollary 11, $(\alpha, \beta) \in \Upsilon_{\mu, \rho}$. Now, we consider the case where $\mu<0$. From the previous discussions, Proposition 7 and Theorem 8, we can be sure that $\Phi_{\mu, \rho}=\Theta_{\mu, \rho}$. Next, we claim that $\Omega_{\mu, \rho}=\left\{\mathscr{H}_{-\mu, \rho}^{\prime \prime} \backslash \partial \mathscr{H}_{-\mu, \rho}^{\prime \prime}\right\}$, where $\mathscr{H}_{-\mu, \rho}$ is given by (75). From the previous discussions and Corollary 22, we can be sure that $\varphi$ is a positive solution if and only if $\mathscr{H}_{-\mu, \rho} \backslash \partial \mathscr{H}_{-\mu, \rho}$. Thus, from Proposition 7 and Theorem 8, it follows that $\Omega_{\mu, \rho}=\left\{\mathscr{H}_{-\mu, \rho}^{\prime \prime} \backslash \partial \mathscr{H}_{-\mu, \rho}^{\prime \prime}\right\}$ and this completes the proof.

Corollary 26. Let $\varphi=\left\{\varphi_{k}\right\}_{k \in \mathbf{Z}}$ be a solution of (7) with $|\rho|>$ 2. $\varphi$ is a nonnegative and periodic solution if and only if $\mu \geq 0$ and $\left(\varphi_{m}, \varphi_{m+1}\right)=(\mu /(2+\rho), \mu /(2+\rho))$ for some $m \in \mathbf{Z} ; \varphi$ is a negative and periodic solution if and only if $\mu<0$ and $\left(\varphi_{n}, \varphi_{n+1}\right)=(\mu /(2+\rho), \mu /(2+\rho))$ for some $n \in \mathbf{Z}$.

From Proposition 2, Theorem 10, Corollary 11, and Theorems 23,24 , and 25 , we can easily see that the statement of Corollary 26 holds.

This section shows that both $\Phi_{\mu, \rho}$ and $\Omega_{\mu, \rho}$ are nonempty and if $\varphi$ is not a constant solution of (7) with $|\mu|>2$, then $\varphi$ is not periodic. Next, we will discuss $\Phi_{\mu, \rho}$ and $\Omega_{\mu, \rho}$ where $|\rho|=2$.

\section{Nonnegative and Negative Solutions when $|\rho|=2$}

Let $\varphi=\left\{\varphi_{k}\right\}_{k \in \mathbf{Z}}$ be a solution of (7) with $\rho=|2|$ and $\left(\varphi_{0}, \varphi_{1}\right)=$ $(\alpha, \beta)$. Let $f=\Gamma_{\mu, \rho}(\alpha, \beta)$. Recall that $\mathcal{O}(\varphi)$ is on the conic section $\Gamma_{\mu, \rho, f}$ which is a parabola or degenerate parabola that is made up of either one line or two distinct parallel lines. 
In this section, we now discuss the nonnegative and negative solutions of ( 7 ) by investigating $\rho=2$ and $\rho=-2$.

Theorem 27. Let $\varphi=\left\{\varphi_{k}\right\}_{k \in \mathbf{Z}}$ be a solution of (7) with $\rho=2$. Suppose $\left(\varphi_{m}, \varphi_{m+1}\right)=(\alpha, \beta)$ for some $m \in \mathbf{Z}$.

(i) Suppose $\mu \geq 0$. Then $\varphi$ cannot be a negative solution; furthermore, $\varphi$ is a nonnegative solution if and only if

$$
(\alpha, \beta) \in\left\{(x, y) \in \bar{L}_{\mu, 2,-\mu^{2} / 4}^{+} \mid x, y \geq 0\right\} .
$$

(ii) Suppose $\mu<0$. Then $\varphi$ cannot be a nonnegative solution; furthermore, $\varphi$ is a negative solution if and only if

$$
(\alpha, \beta) \in\left\{(x, y) \in \bar{L}_{\mu, 2,-\mu^{2} / 4}^{-} \mid x, y<0\right\} .
$$

Proof. From the previous discussions, $\Gamma_{\mu, 2, f}$, where $f=$ $\Gamma_{\mu, 2}(\alpha, \beta) \geq-\mu^{2} / 4$, is a degenerate parabola made up of two lines, $\bar{L}_{\mu, 2, f}^{+}$and $\bar{L}_{\mu, 2, f}^{-}$defined by (24) and (25), respectively. Moreover, if $f=-\mu^{2} / 4$, then $\bar{L}_{\mu, 2, f}^{+}=\bar{L}_{\mu, 2, f}^{-}$; otherwise, $\bar{L}_{\mu, 2, f}^{+}$ and $\bar{L}_{\mu, 2, f}^{-}$are two distinct parallel lines. We first consider the case where $\mu \geq 0$. By Theorem 12 and the Tracking Procedure, if $\alpha, \beta \geq 0$ such that $f=\Gamma_{\mu, 2}(\alpha, \beta)=-\mu^{2} / 4$, then $\varphi$ is periodic with period 2 and $\varphi_{2} \geq 0$ which implies $\varphi$ is a nonnegative solution. Conversely, if $\varphi$ is a nonnegative solution, then $\alpha, \beta \geq 0$ and by Theorem 12, $f=-\mu^{2} / 4$ which implies $(\alpha$, $\beta) \in \bar{L}_{\mu, 2,-\mu^{2} / 4}^{+}=\bar{L}_{\mu, 2,-\mu^{2} / 4}^{-}$. Accordingly, we see that

$$
\Phi_{\mu, 2}=\left\{(x, y) \in \bar{L}_{\mu, 2,-\mu^{2} / 4}^{+} \mid x, y \geq 0\right\} .
$$

On the other hand, by Theorem 12 again, we can be sure that $\varphi$ cannot be a nonpositive solution which implies $\Omega_{\mu, \rho}$ is empty. Next, we suppose $\mu<0$. From the previous discussions and Proposition 7, if $\varphi$ is a nonnegative solution then $-\varphi$ is a solution of (7) with $-\mu>0$ which is not possible. Also, $\bar{L}_{\mu, 2,-\mu^{2} / 4}^{+}$and $\bar{L}_{\mu, 2,-\mu^{2} / 4}^{-}$are symmetric with respect to line $x+y=0$ and from Proposition 7 and Theorem 8, it follows that

$$
\Omega_{\mu, \rho}=\left\{(x, y) \in \bar{L}_{\mu, 2,-\mu^{2} / 4}^{-} \mid x, y<0\right\}
$$

and this completes the proof.

Theorem 28. Let $\varphi=\left\{\varphi_{k}\right\}_{k \in \mathbf{Z}}$ be a solution of (7) with $\rho=-2$. Suppose $\left(\varphi_{m}, \varphi_{m+1}\right)=(\alpha, \beta)$ for some $m \in \mathbf{Z}$.

(i) Suppose $\mu=0$. Then $\varphi$ is a nonnegative solution if and only if $\alpha, \beta \geq 0$ and $(\alpha, \beta) \in \bar{\Pi}$ which is the $x=y$ line; furthermore, $\varphi$ is negative solution if and only if $(\alpha, \beta) \in \bar{\Pi}$ and $\alpha, \beta<0$.

(ii) Suppose $\mu>0$. Then $\varphi$ cannot be a negative solution; furthermore, $\varphi$ is a nonnegative solution if and only if $(\alpha, \beta) \in \mathscr{H}_{\mu,-2}$ which is the intersection of the closed half planes defined by (75).

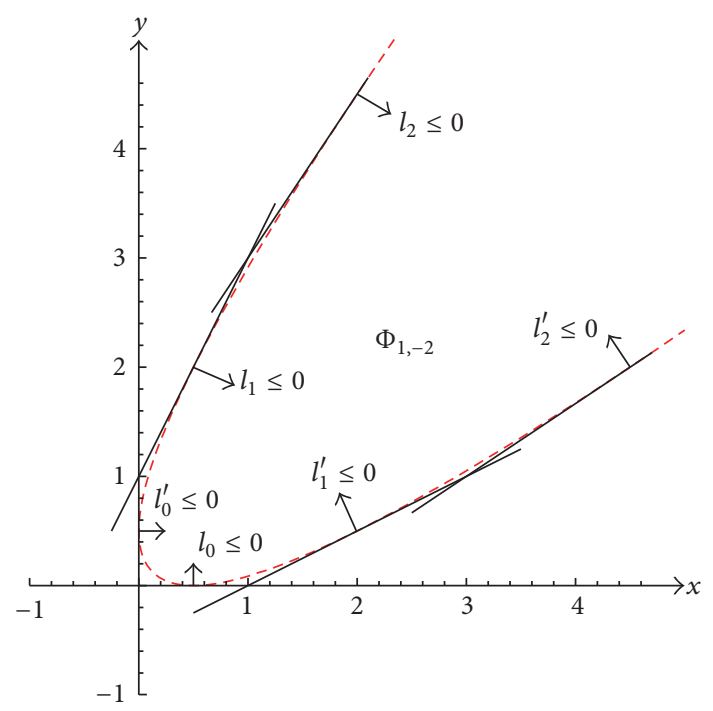

FIGURE 19: The set $\Phi_{1},-2$.

(iii) Suppose $\mu<0$. Then $\varphi$ cannot be a nonnegative solution; furthermore, $\varphi$ is a negative solution if and only if

$$
(\alpha, \beta) \in\left\{\mathscr{H}_{-\mu,-2}^{\prime \prime} \backslash \partial \mathscr{H}_{-\mu,-2}^{\prime \prime}\right\}
$$

where $\mathscr{H}_{-\mu,-2}$ is the intersection of the closed half planes defined by (75).

Proof. Recall that $\bar{\Pi}$ is the line $x=y$ which is one principal axis of $\Gamma_{\mu, \rho, f}$, where $f=\Gamma_{\mu, \rho}(\alpha, \beta)$. Suppose $\mu=0$. By Theorem 13, if $(\alpha, \beta) \notin \bar{\Pi}$, then $\varphi$ is neither a nonnegative nor a nonpositive solution. If $(\alpha, \beta) \in \bar{\Pi}$, then $\varphi$ is 1 -periodic and we see that (i) holds by direct verification. Suppose $\mu>0$. Then by Theorem 21, we can be sure that $\Phi_{\mu,-2}=\mathscr{H}_{\mu,-2}$ as required and $\mathscr{H}_{\mu,-2}$ is depicted in Figure 19 . For the case of (iii), from Proposition 7 and Theorem 8, it follows that (87) is true and this completes the proof.

In view of Theorem 28. we have shown that $\Phi_{\mu,-2}=$ $\mathscr{H}_{\mu,-2}$ which is the intersection of the half planes defined by the tangent lines at all the points of $\mathcal{O}\left({ }_{\rho}^{\mu} \chi\right)$ on the parabola $\Gamma_{\mu,-2,-\mu^{2} / 4}$. Figure 19 illustrates $\Phi_{1,-2}$ where the red-dashed curve is the parabola $\Gamma_{1,-2,-1 / 4}$. The discussions of the cases where $|\rho| \geq 2$ are done and move forward to analyze the last cases where $|\rho|<2$.

\section{Nonnegative and Negative Solutions when $|\rho|<2$}

In the previous discussions, we have analyzed the cases where $|\rho| \geq 2$ and derived the necessary and sufficient conditions for a solution $\varphi$ to be nonnegative (or negative). Let $\varphi=\left\{\varphi_{k}\right\}_{k \in \mathbf{Z}}$ be a solution of (7). From our analyses of the case where $|\rho| \geq 2$, the periodicity of $\varphi$ has no significant role in the analysis (albeit $\varphi$ may be periodic with least period $\omega \leq 2$ ); yet the periodicity of $\varphi$ is of great importance when discussing 
the case where $|\rho|<2$. In Corollary 16, we illustrated the necessary and sufficient conditions for $\varphi$ to be a periodic solution of (7) with $|\rho|<2$ and we will discuss the case where $\mu=0, \mu>0$, and $\mu<0$ in the sequel.

Theorem 29. Let $\varphi=\left\{\varphi_{k}\right\}_{k \in \mathbf{Z}}$ be a solution of (7) with $|\rho|<2$ and $\mu=0$. Then $\varphi$ cannot be a negative solution; furthermore, $\varphi$ is a nonnegative solution if and only if $\varphi$ is the null solution.

Proof. Let $\left(\varphi_{0}, \varphi_{1}\right)=(\alpha, \beta)$ such that $f=\Gamma_{0, \rho}(\alpha, \beta)$. Let $\left\{\left(x_{k}, y_{k}\right)\right\}_{k \in \mathbf{N}}$ be generated by the Tracking Procedure with input $\left(x_{0}, y_{0}\right)=(\alpha, \beta)$. Suppose $(\alpha, \beta)=(0,0)$. From the previous discussions, $f=\Gamma_{0, \rho}(0,0)=0$ which implies $\Gamma_{0, \rho, 0}$ is a point ellipse. On the other hand, by Proposition $2,\left(\varphi_{0}, \varphi_{1}\right)=$ $(0,0)$ which leads to $\varphi_{k}=0$, for all $k \in \mathbf{N}$ and, thus, $\varphi$ is a nonnegative solution with least period 1 . Now, we suppose $(\alpha, \beta) \neq(0,0)$. Then $\Gamma_{0, \rho, f}$ is an ellipse and we show that $\varphi$ is neither a nonnegative nor a negative solution. To this end, we consider $0 \leq \rho<2$ and $-2<\rho<0$. Suppose $0 \leq \rho<2$. By Proposition 6, $\varphi$ cannot be a nonpositive solution which implies $\varphi$ is not negative. If $\varphi$ is a nonnegative solution, then by Proposition $7,-\varphi$ is a nonpositive solution which is a contradiction. The case where $-2<\rho<0$ can be handled similarly and this completes the proof.

Next, we consider the corresponding results when $|\rho|<2$ and $\mu \neq 0$.

Theorem 30. Let $\varphi=\left\{\varphi_{k}\right\}_{k \in \mathbf{Z}}$ be a solution of (7) with $|\rho|<2$ and $\mu \neq 0$. Suppose $\pi^{-1} \arccos (-\rho / 2) \notin \mathbf{Q}$ and let $\left(\varphi_{m}\right.$, $\left.\varphi_{m+1}\right)=(\alpha, \beta)$ for some $m \in \mathbf{Z}$.

(i) Suppose $\mu>0$. Then $\varphi$ cannot be a negative solution; furthermore, $\varphi$ is a nonnegative solution if and only if

$$
(\alpha, \beta) \in\left\{(x, y) \in \mathbf{R}^{2} \mid \Gamma_{\mu, \rho}(x, y) \leq \frac{1}{4} \mu^{2}\right\},
$$

which is the interior of the ellipse $\Gamma_{\mu, \rho,-\mu^{2} / 4}$.

(ii) Suppose $\mu<0$. Then $\varphi$ cannot be a nonnegative solution; furthermore, $\varphi$ is a negative solution if and only if $(\alpha, \beta) \in \Lambda_{-\mu, \rho}^{\prime \prime}$, where

$\Lambda_{-\mu, \rho}$

$$
=\left\{(x, y) \in \mathbf{R}^{2} \mid \Gamma_{-\mu, \rho}(x, y) \leq \frac{1}{4} \mu^{2}\right\} \backslash\left\{\mathcal{O}\left(\begin{array}{l}
\mu \\
\rho
\end{array}\right)\right\} .
$$

Proof. Without loss of generality, we let $m=0$. Also, for the sake of convenience, let $P_{c}=(\mu /(2+\rho), \mu /(2+\rho))$ and $r=\pi^{-1} \arccos (-\rho / 2)$. Also, let $\mathcal{O}^{+}(\varphi)=\left\{\left(x_{k}, y_{k}\right)\right\}_{k \in \mathbf{N}}$ be generated by the Tracking Procedure with input $\left(x_{0}, y_{0}\right)=$ $(\alpha, \beta)$. Then we consider two cases where $\mu>0$ and $\mu<$ 0 . Here, if $(\alpha, \beta)=P_{c}$, then, by the previous discussions, $\Gamma_{\mu, \rho,-\mu^{2} /(2+\rho)}$ is a point ellipse so that $\left(x_{k}, y_{k}\right)=P_{c}$, for all $k \in \mathbf{N}$. Note that $\Gamma_{\mu, \rho,-\mu^{2} / 4}$ is tangent to lines $x=0$ and $y=0$ at $(0, \mu / 2)$ and $(\mu / 2,0)$, respectively. Suppose $\mu>0$. We first show that $\varphi$ cannot be a nonpositive solution. If $\rho \geq 0$, then, by Proposition 6, $\varphi$ is not a nonpositive solution. If $\varphi$ is nonpositive with $\rho<0$, then, by the previous discussions, $(\alpha, \beta) \neq P_{c}$ and by Corollary 18 , there is some $m \in \mathbf{N}$ such that $x_{m} \geq 0$ or $y_{m} \geq 0$. Furthermore, if $\alpha=\beta=0$, then, by (7), $\varphi_{2}=\mu>0$. Accordingly, $\varphi$ is not a nonpositive solution when $\mu>0$.

Now, we show

$$
\Phi_{\mu, \rho}=\left\{(x, y) \in \mathbf{R}^{2} \mid \Gamma_{\mu, \rho}(x, y) \leq \frac{1}{4} \mu^{2}\right\},
$$

where $\rho>0$. Note that $s, t \geq 0$, where $(s, t) \in\left\{(x, y) \in \mathbf{R}^{2} \mid\right.$ $\left.\Gamma_{\mu, \rho}(x, y) \leq(1 / 4) \mu^{2}\right\}$. If (88) holds, then, by Theorem 8 , we can be sure that $\Gamma_{\mu, \rho, f} \subseteq \Phi_{\mu, \rho}$, where $f=\Gamma_{\mu, \rho}(\alpha, \beta)$. Accordingly, $\varphi_{m} \geq 0$ for all $m \in \mathbf{Z}$. On the other hand, suppose (88) does not hold. Since $r \notin \mathbf{Q}$, by Theorem 17, there is some $i \in \mathbf{N}$ such that $\bar{l}_{i}(\alpha, \beta)>0$ (or some $j \in \mathbf{N}$ such that $\bar{l}_{j}^{\prime}(\alpha, \beta)>$ $0)$ which implies $\varphi$ is not nonnegative. Hence, (90) is true. Next, suppose $\mu<0$. First of all, by the previous discussions and Corollary 22, we see that $\varphi$ is a positive solution if and only if $(\alpha, \beta) \in \Lambda_{-\mu, \rho}$. Thus, from Proposition 7 and Theorem 8 , it follows that $\Omega_{\mu, \rho}=\Lambda_{-\mu, \rho}^{\prime \prime}$ as required.

The proof is complete.

Theorems 30 and 17 and Proposition 7 help us to analyze the necessary and sufficient conditions for an aperiodic solution $\varphi$ of (7) to be nonnegative (or negative). For the case when $\varphi$ is periodic, we have to resort to the help of the specific solution ${ }_{\rho}^{\mu} \chi$ of (7).

Theorem 31. Let $\varphi=\left\{\varphi_{k}\right\}_{k \in \mathbf{Z}}$ be a solution of (7) with $|\rho|<2$ and $\mu \neq 0$. Suppose $\pi^{-1} \arccos (-\rho / 2) \in \mathbf{Q}$ and $\left(\varphi_{m}, \varphi_{m+1}\right)=$ $(\alpha, \beta)$, for some $m \in \mathbf{Z}$. Then $\varphi$ is periodic and we have the following.

(i) Suppose $\mu>0$. Then $\varphi$ cannot be a negative solution; furthermore, $\varphi$ is a nonnegative solution if and only if $(\alpha, \beta) \in \mathscr{H}_{\mu, \rho}$ which is the intersection of the closed half planes defined by (75).

(ii) Suppose $\mu<0$. Then $\varphi$ cannot be a nonnegative solution; furthermore, $\varphi$ is a negative solution if and only if

$$
(\alpha, \beta) \in\left\{\mathscr{H}_{-\mu, \rho}^{\prime \prime} \backslash \partial \mathscr{H}_{-\mu, \rho}^{\prime \prime}\right\}
$$

where $\mathscr{H}_{-\mu, \rho}$ is the intersection of the closed half planes defined by (75).

Proof. Without loss of generality, we let $m=0$. Also, let $\mathcal{O}(\varphi)=\left\{\left(x_{k}, y_{k}\right)\right\}_{k \in \mathbf{N}}$ be generated by the Tracking Procedure with input $\left(x_{0}, y_{0}\right)=(\alpha, \beta)$. Suppose $\mu>0$. From the previous discussions, if $(\alpha, \beta)=(\mu /(2+\rho), \mu /(2+\rho))$, then $\varphi$ is a nonnegative solution with least period 1 . If $(\alpha, \beta) \neq$ $(\mu /(2+\rho), \mu /(2+\rho))$, then, by Corollary $16, \varphi$ is periodic and $\Gamma_{\mu, \rho, f}$ is an ellipse, where $f=\Gamma_{\mu, \rho}(\alpha, \beta)$. Also, by Theorem 21, we can be sure that $\Phi_{\mu, \rho}=\mathscr{H}_{\mu, \rho}$ as desired. Next, we show that $\varphi$ is not a nonpositive solution. First of all, by Proposition 6, $\varphi$ is not nonpositive when $\rho \geq 0$. Secondly, suppose $\varphi$ is a nonpositive solution of (7) with $\rho<0$. Then by Corollary 18, there is some $m \in \mathbf{N}$ such that $x_{m}$ or $y_{m} \geq 0$. If $x_{m}=$ 
$y_{m}=0$, then, by (7), $x_{m+1}=\mu>0$ which is a contradiction. Accordingly, $\varphi$ is not a nonpositive solution.

Suppose $\mu<0$. By Corollary 22, Proposition 7, and Theorem 8, it follows that (91) holds. Also, if $\varphi$ is a nonnegative solution of (7) with $\mu<0$, then, by Proposition 7, $-\varphi$ is a solution of (7) with $-\mu>0$ which is not possible and this completes the proof.

Corollary 32. Let $\varphi=\left\{\varphi_{k}\right\}_{k \in \mathbf{Z}}$ be a solution of (7) with $|\rho|<2$ and $\mu>0$. Suppose $r=\pi^{-1} \arccos (-\rho / 2)=(n / m) \in \mathbf{Q}$ such that $0<r<1$ and $r$ is irreducible. Then $\Phi_{\mu, \rho}$ is a closed polygon with $\omega$ sides which is circumscribed around the ellipse $\Gamma_{\mu, \rho,-\mu^{2} / 4}$ and $\omega$ is either $2 n$ or $n$ depending on whether $m$ is even or odd, respectively.

Proof. In view of Theorem 21, $\Phi_{\mu, \rho}=\mathscr{H}_{\mu, \rho}$ which is defined by the tangent lines of $\Gamma_{\mu, \rho,-\mu^{2} / 4}$. Furthermore, all the lines are tangent to $\Gamma_{\mu, \rho,-\mu^{2} / 4}$ at the points which are generated by a specific solution ${ }_{\rho}^{\mu} \chi$ of $(7)$ with $\left({ }_{\rho}^{\mu} \chi_{0},{ }_{\rho}^{\mu} \chi_{1}\right)=(\mu / 2,0)$. Since $\pi^{-1} \arccos (-\rho / 2) \in \mathbf{Q}$, by Corollary $16,{ }_{\rho}^{\mu} \chi$ is $\omega$-periodic where $\omega$ is dependent on $n$ and the parity of $m$. Note that $\omega>2$. Accordingly, we see that $\Phi_{\mu, \rho}$ is defined by $\omega$ distinct lines. To see $\Phi_{\mu, \rho}$ is a closed polygon with $\omega$ sides which is circumscribed around $\Gamma_{\mu, \rho,-\mu^{2} / 4}$, it is sufficient to show that $\Phi_{\mu, \rho}$ is closed. If $\rho=0$, then ${ }_{\rho}^{\mu} \chi$ is 4-periodic such that

$$
\Phi_{\mu, \rho} \subset\left\{(x, y) \in \mathbf{R}^{2} \mid 0 \leq x \leq \mu, 0 \leq y \leq \mu\right\} ;
$$

if $0<\rho<2$, then, by (70), $h_{\mu, \rho, 2}(x, y)=\mu-\rho x-y$ such that

$$
\begin{aligned}
& \Phi_{\mu, \rho} \\
& \quad \subset\left\{(x, y) \in \mathbf{R}^{2} \mid \mu-\rho x-y \geq 0, x \geq 0, y \geq 0\right\} .
\end{aligned}
$$

Accordingly, $\Phi_{\mu, \rho}$ is closed, where $0 \leq \rho<2$. Suppose $-2<\rho<0$. To see $\Phi_{\mu, \rho}$ is closed, it is sufficient to show that there is some $i \in \mathbf{N}$ such that $\Pi_{\perp}\left({ }_{\rho}^{\mu} \chi_{i}{ }^{\mu}{ }_{\rho}^{\mu} \chi_{i+1}\right)>0$. Since $\Pi_{\perp}\left({ }_{\rho}^{\mu} \chi_{0},{ }_{\rho}^{\mu} \chi_{1}\right)<0$, in view of Corollary 18, there is some $j \in \mathbf{N}$ such that $\Pi_{\perp}\left({ }_{\rho}^{\mu} \chi_{j},{ }_{\rho}^{\mu} \chi_{j+1}\right)>0$ as required and this completes the proof.

Corollary 32 gives an explicit description of $\Phi_{\mu, \rho}$, where $|\rho|<0$, and two examples for the cases $(\rho, \mu)=(1,1)$ and $(\rho, \mu)=(-1,1)$ are in Figures 20 and 21 respectively.

\section{Conclusions}

In the previous sections, we have analyzed the nonnegative (or negative) solution $\varphi=\left\{\varphi_{k}\right\}_{k \in \mathbf{Z}}$ of (7) by investigating the pairs $\left(\varphi_{m}, \varphi_{m+1}\right)$ for all $m \in \mathbf{Z}$ which are on a conic section (or degenerate one). Specifically, the orbit $\mathcal{O}(\varphi)$ is the collection of $\left(\varphi_{m}, \varphi_{m+1}\right)$ for all $m \in \mathbf{Z}$ which is a subset of a conic section and by the Tracking Procedure, $\left(\varphi_{n+1}, \varphi_{n+2}\right)$ is uniquely determined by giving $\left(\varphi_{n}, \varphi_{n+1}\right)$. By means of this geometric method, many qualitative properties of (7) can be obtained. In particular, we obtain the necessary and sufficient conditions for $\varphi$ to be a nonnegative (or negative) solution.

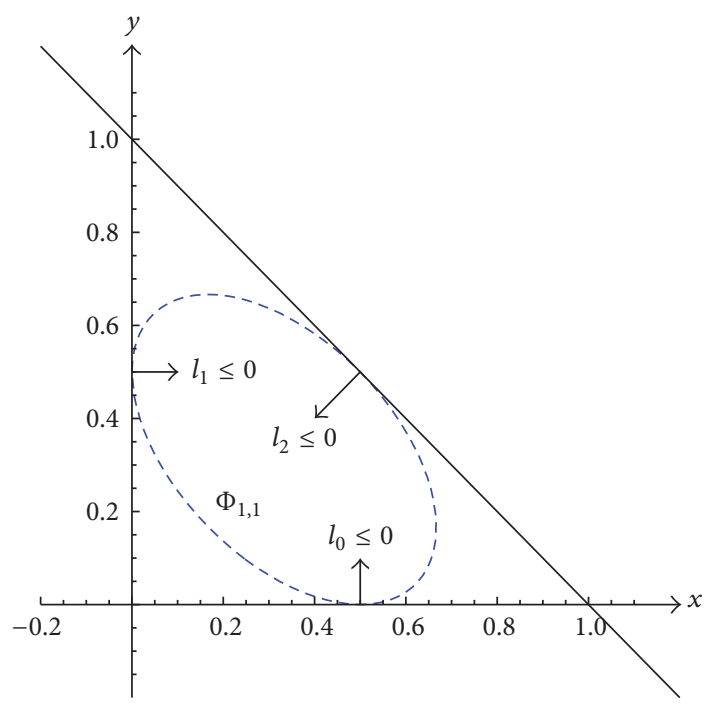

Figure 20: The set $\Phi_{1,1}$.

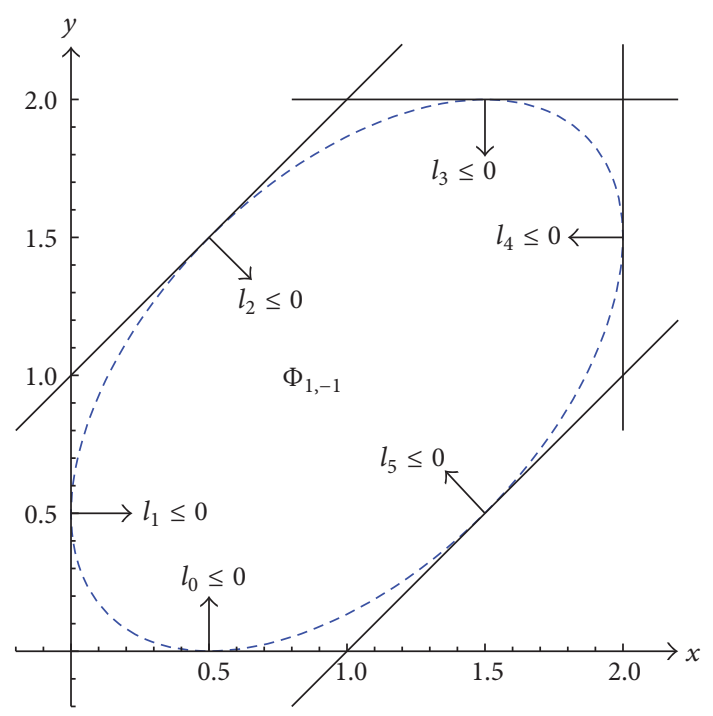

Figure 21: The set $\Phi_{1,-1}$.

Now, we summarize the necessary and sufficient conditions for $\varphi$ to be a nonnegative (or negative) periodic solution of (7) as follows (recall that for a set $S \subset \mathbf{R}^{2}, S^{\prime \prime}$ is the set on $\mathbf{R}^{2}$ such that $S$ and $S^{\prime \prime}$ are symmetric with respect to the $x+y=0$ line).

(1) Suppose $|\rho|>2 . \varphi$ is a nonnegative and periodic solution if and only if $\rho \mu \geq 0$, and for some $i \in \mathbf{Z}$, $\left(\varphi_{i}, \varphi_{i+1}\right)=(\mu /(2+\rho), \mu /(2+\rho))$. Furthermore, such $\varphi$ must satisfy $\varphi_{j}=\mu /(2+\rho)$ for all $j \in \mathbf{Z}$ (and hence is 1-periodic).

(2) Suppose $|\rho|<2 . \varphi$ is a nonnegative and periodic solution if and only if $\mu>0$

$$
\frac{1}{\pi} \arccos \left(-\frac{1}{2} \rho\right)=\frac{m}{n} \in \mathbf{Q},
$$


(where $m / n$ is irreducible and $0<m<n$ ) and for some $i \in \mathbf{Z},\left(\varphi_{i}, \varphi_{i+1}\right) \in \mathscr{H}_{\mu, \rho}$ which, by Corollary 32 , is a closed $\omega$-side polygon where $\omega$ is either $n$ or $2 n$ depending on whether $m$ is even or odd, respectively. Furthermore, such $\varphi$ must either satisfy $\left(\varphi_{j}, \varphi_{j+1}\right)=$ $(\mu /(2+\rho), \mu /(2+\rho))$ for all $j \in \mathbf{Z}$ (and hence is 1 periodic) or $\varphi$ is $\omega$-periodic.

(3) Suppose $|\rho|=2$. If $\rho=2$, then $\varphi$ is a nonnegative and periodic solution if and only if $\mu \geq 0$, and for some $m \in \mathbf{Z}, \varphi_{m}, \varphi_{m+1} \geq 0$ and $\left(\varphi_{m}, \varphi_{m+1}\right)$ is on the $x+y=\mu / 4$ line; if $\rho=-2$, then $\varphi$ is a nonnegative and periodic solution if and only if $\mu=0$, and for some $n \in \mathbf{Z}, \varphi_{n}, \varphi_{n+1} \geq 0$, and $\left(\varphi_{n}, \varphi_{n+1}\right)$ lie on the $x=y$ line. Furthermore, such $\varphi$ is either 1-periodic if $\varphi_{m}=\varphi_{m+1}\left(\right.$ and 2-periodic if $\left.\varphi_{m} \neq \varphi_{m+1}\right)$ when $\rho=2$ or 1-periodic when $\rho=-2$.

As for the negative periodic solutions, we have the following.

(1) Suppose $|\rho|>2$. $\varphi$ is a negative and periodic solution if and only if $\rho \mu<0$ and for some $i \in \mathbf{Z},\left(\varphi_{i}, \varphi_{i+1}\right)=$ $(\mu /(2+\rho), \mu /(2+\rho))$. Furthermore, such $\varphi$ must satisfy $\varphi_{j}=\mu /(2+\rho)$ for all $j \in \mathbf{Z}$ (and hence is 1-periodic).

(2) Suppose $|\rho|<2$. $\varphi$ is a negative and periodic solution if and only if $\mu<0$

$$
\frac{1}{\pi} \arccos \left(-\frac{1}{2} \rho\right)=\frac{m}{n} \in \mathbf{Q},
$$

(where $m / n$ is irreducible and $0<m<n$ ) and for some $i \in \mathbf{Z},\left(\varphi_{i}, \varphi_{i+1}\right) \in\left\{\mathscr{H}_{-\mu, \rho}^{\prime \prime} \backslash \partial \mathscr{H}_{-\mu, \rho}^{\prime \prime}\right\}$, where by Corollary $32, \mathscr{H}_{-\mu, \rho}$ is a closed $\omega$-side polygon and $\omega$ is either $n$ or $2 n$ depending on whether $m$ is even or odd, respectively. Furthermore, such $\varphi$ must either satisfy $\left(\varphi_{j}, \varphi_{j+1}\right)=(\mu /(2+\rho), \mu /(2+\rho))$ for all $j \in \mathbf{Z}$ (i.e., $\varphi$ is 1 -periodic) or $\varphi$ is $\omega$-periodic.

(3) Suppose $|\rho|=2$. If $\rho=2$, then $\varphi$ is a negative and periodic solution if and only if $\mu<0$, and for some $m \in \mathrm{Z}, \varphi_{m}, \varphi_{m+1}<0$ and $\left(\varphi_{m}, \varphi_{m+1}\right)$ is on the $x+y=\mu / 4$ line; if $\rho=-2$, then $\varphi$ is a negative and periodic solution if and only if $\mu=0$, and for some $n \in \mathrm{Z}, \varphi_{n}, \varphi_{n+1}<0$ and $\left(\varphi_{n}, \varphi_{n+1}\right)$ lie on the $x=y$ line. Furthermore, such $\varphi$ is either 1-periodic if $\varphi_{m}=\varphi_{m+1}\left(\right.$ and 2-periodic if $\left.\varphi_{m} \neq \varphi_{m+1}\right)$ when $\rho=2$ or 1-periodic when $\rho=-2$.

With the help of the Tracking Procedure, the behaviors of the solution $\varphi$ of (7) can be visualized (instead of investigating each term of $\varphi$ by the method of general solutions). By means of our geometric method, we may save lots of calculation and most properties of $\varphi$ which may be obtained by the general solutions are also available from our geometric discoveries.

In [1-3], we conducted complete analyses on the periodic solutions (7) with $\mu=1$ and $\rho \in\{-1,0,1\}$. By means of breaking a solution $\psi$ into two subsequences, the companion sequence and the error sequence, we successfully show that both the companion sequence and error sequence are periodic so that $\psi$ is periodic. Note that for $\rho=-1,0$, and 1 , we have $\arccos (-\rho / 2)=\pi / 3, \pi / 2$, and $2 \pi / 3$, respectively.
The nice thing here is that by means of our geometric method, not only can we track the orbit of a solution $\varphi$ on $\mathbf{R}^{2}$ easily but also we may obtain the conditions for $\varphi$ to be nonnegative (or negative) quickly (especially for the case where $|\rho|<2$ and $\varphi$ is periodic). By [1-3], we have observed the importance of nonnegative solutions of (7) with $\rho=0,1$, and -1 , respectively. However, we also realize the difficulties of analyzing the solution $\zeta$ of (7) with $\rho \notin\{-1,0,1\}$ since the breaking of $\zeta$ into companion and error sequences is not applicable for all the solutions anymore.

Finally, in this paper we have only touched upon one class of steady state solutions of our original neural network. Much work has to be done before the dynamic behaviors of its solutions can be fully understood. Our contributions here, however, are that elementary geometric methods can be used to explain some of the complex phenomena obtained from simulations (see, e.g., Figures 1 and 2) and these methods are also expected to be applied in other piecewise smooth dynamic systems.

\section{Conflicts of Interest}

The authors declare that there are no conflicts of interest whatsoever regarding the publication of this paper.

\section{Acknowledgments}

The third author is partially supported by the Ministry of Science and Technology, R.O.C. under Grant MOST 104-2221E-007-061-MY3 which also complies with the declaration of conflicts of interest stated above.

\section{References}

[1] Y. C. Chang, G.-Q. Wang, and S. S. Cheng, "Complete set of periodic solutions of a discontinuous recurrence equation," Journal of Difference Equations and Applications, vol. 18, no. 7, pp. 1133-1162, 2012.

[2] Y. C. Chang and S. S. Cheng, "Complete periodic behaviours of real and complex bang bang dynamical systems," Journal of Difference Equations and Applications, vol. 20, no. 5-6, pp. 765810, 2014.

[3] Y. C. Chang and S. S. Cheng, "Complete periodicity analysis for a discontinuous recurrence equation," International Journal of Bifurcation and Chaos, vol. 23, no. 4, Article ID 1330012, 34 pages, 2013.

[4] L. Dou, C. Hou, and S. S. Cheng, "Bifurcation analysis for nonlinear recurrence relations with threshold control and $2 k$ periodic coefficients," Discrete Dynamics in Nature and Society, vol. 2015, Article ID 610345, 13 pages, 2015.

[5] C. Hou and S. S. Cheng, "Limit 2-cycles for a discrete-time bang-bang control model," Discrete Dynamics in Nature and Society, vol. 2012, Article ID 735623, 10 pages, 2012.

[6] C. Hou and S. S. Cheng, "Bifurcation in a discrete-time piecewise constant dynamical system," Discrete Dynamics in Nature and Society, vol. 2013, Article ID 492014, 10 pages, 2013.

[7] R. Abu-Saris, F. Allan, S. S. Cheng, and M. Kulenović, "Bifurcation in deterministic discrete dynamical systems: advances in theory and applications," Discrete Dynamics in Nature and Society, vol. 2015, Article ID 960834, 2 pages, 2015. 
[8] Y. Chen, "All solutions of a class of difference equations are truncated periodic," Applied Mathematics Letters, vol. 15, no. 8, pp. 975-979, 2002.

[9] Z. Yuan, L. Huang, and Y. Chen, "Convergence and periodicity of solutions for a discrete-time network model of two neurons," Mathematical and Computer Modelling, vol. 35, no. 9-10, pp. 941-950, 2002.

[10] H. Sedaghat, Nonlinear Difference Equations Mathematical Modelling: Theory and Applications Theory with Applications to Social Science Models, Kluwer Academic, Dordrecht, The Netherland, 1st edition, 2003.

[11] J. Dennis Lawrence, A Catalog of Special Plane Curves, Dover, Mineola, NY, USA, 1972.

[12] S. J. Miller and R. Takloo-Bighash, An Invitation to Modern Number Theory, Princeton University Press, 2006. 


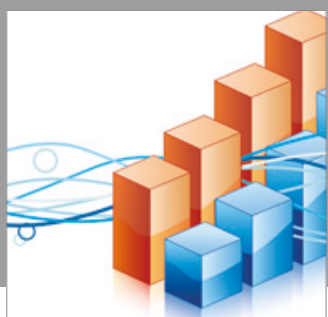

Advances in

Operations Research

vatersals

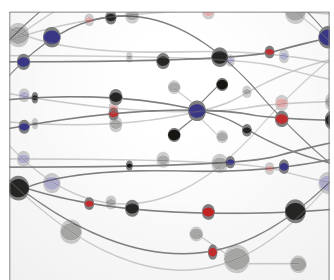

\section{The Scientific} World Journal
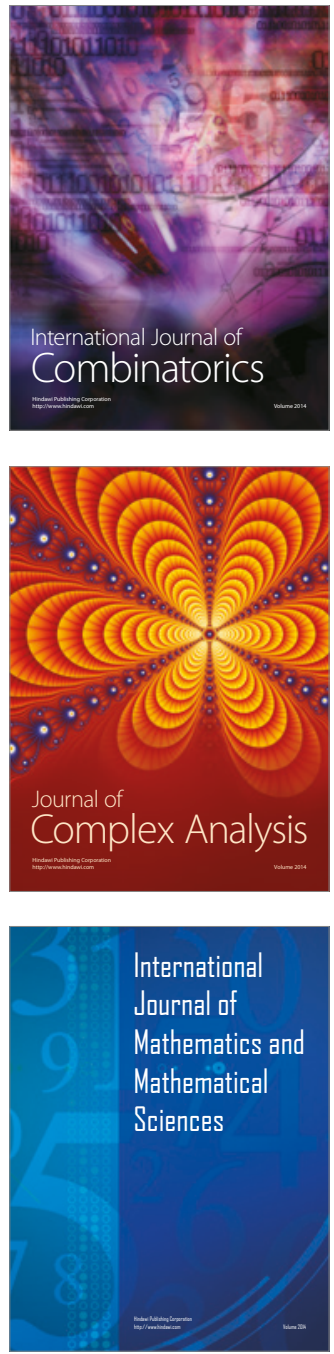
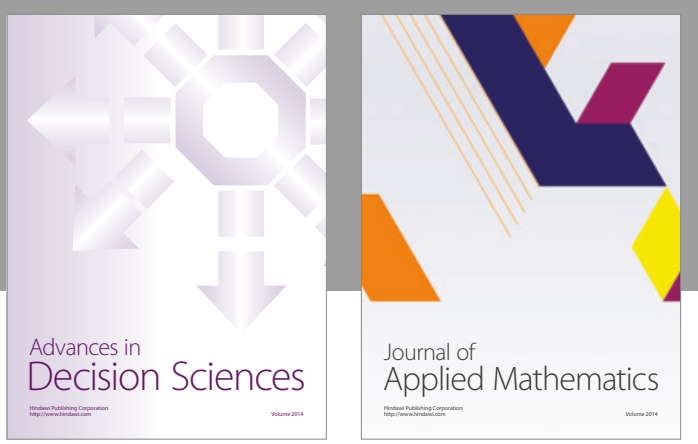

Algebra

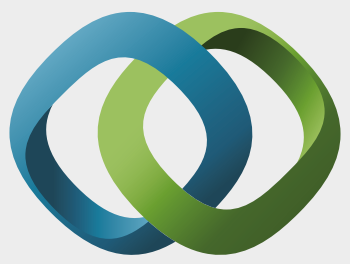

\section{Hindawi}

Submit your manuscripts at

https://www.hindawi.com
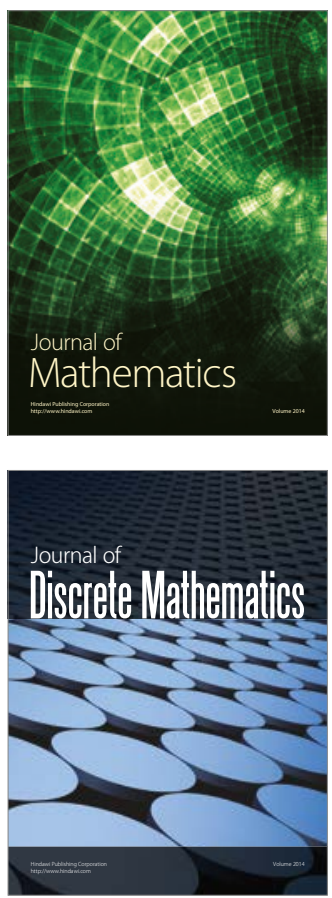

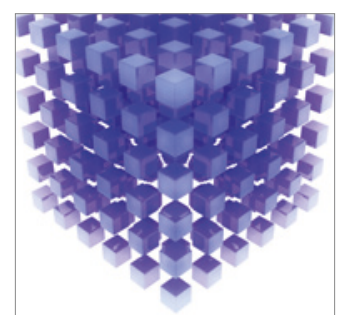

Mathematical Problems in Engineering
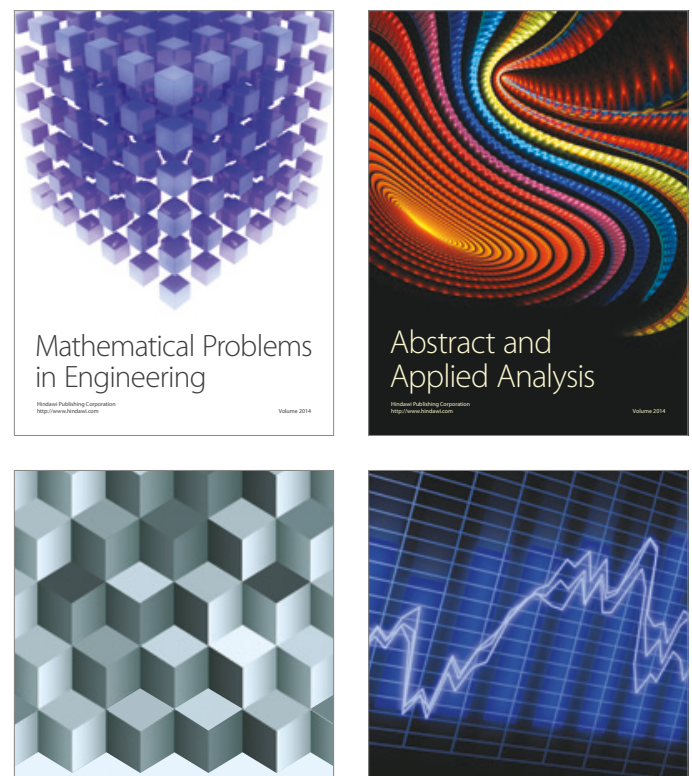

Journal of

Function Spaces

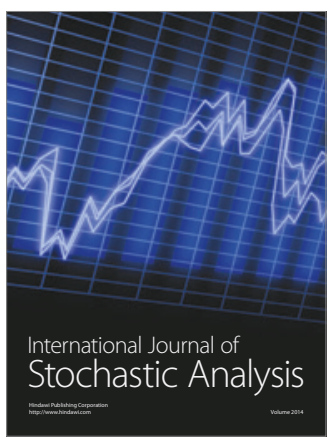

Probability and Statistics
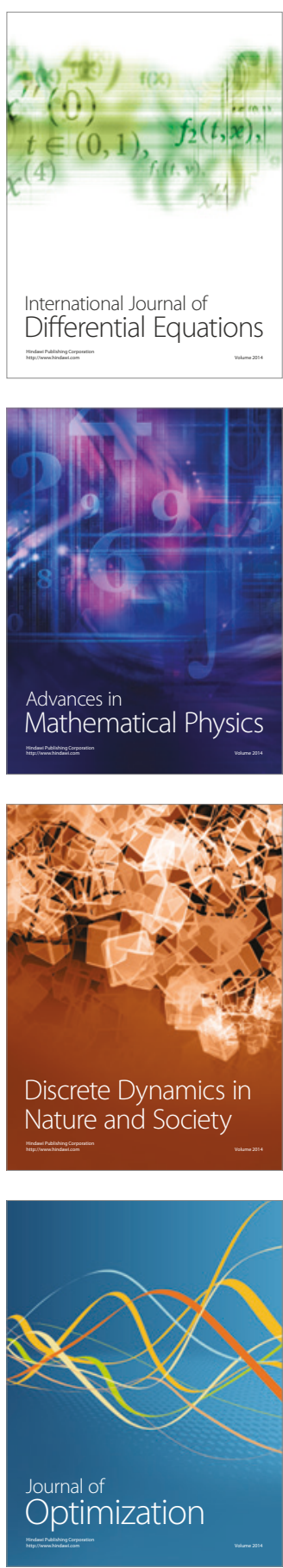